\title{
BAYESIAN GAMES WITH UNAWARENESS AND UNAWARENESS PERFECTION*
}

\author{
${\text { Martin } \text { Meier }^{\dagger} \quad \text { Burkhard C. Schipper }}^{\ddagger}$ \\ This draft: September 12, 2013 \\ First draft: June 6, 2005
}

\begin{abstract}
Applying unawareness belief structures introduced in Heifetz, Meier, and Schipper (2013a), we develop Bayesian games with unawareness, define equilibrium, and prove existence. We show how equilibria are extended naturally from lower to higher awareness levels and restricted from higher to lower awareness levels. We apply Bayesian games with unawareness to investigate the robustness of equilibria to uncertainty about opponents' awareness of actions. We show that a Nash equilibrium of a strategic game is robust to unawareness of actions if and only if it is not weakly dominated. Finally, we discuss the relationship between standard Bayesian games and Bayesian games with unawareness.
\end{abstract}

Keywords: Unawareness, awareness, type-space, incomplete information, Bayesian games, equilibrium, perfection, undominated equilibrium, weak dominance, admissibility, inattention.

JEL-Classifications: C70, C72, D80, D82.

*We thank Aviad Heifetz for substantial input. Moreover, we thank three anonymous reviewers whose comments helped us to improve the manuscript. Martin acknowledges financial support from the Spanish Ministerio de Educación y Ciencia via a Ramon y Cajal Fellowship and Research Grant SEJ2004-07861, and the Fundación Ramón Areces. Burkhard received financial support from the NSF SES-0647811, DFG SFB/TR 15, Minerva Stiftung, and IGA-UCD. We thank Leandro Chaves Rêgo, Eddie Dekel, Enrique Kawamura, Li Jing, Salvatore Modica, Klaus Nehring and participants in the Workshop on Unawareness at Stanford University, 2006, NASEM 2006, LOFT 2006, LAMES 2006, SAET 2007, TARK 2007, Stony Brook 2007, ESEM 2007, NBER GE conference 2007, GAMES 2008 and seminars at Bocconi, UCLA, St. Louis, Tucson, and Venice for helpful comments. Some of the material was circulated previously in a working paper titled "Unawareness, Beliefs and Games".

†Institut für Höhere Studien, Wien. Email: martin.meier@ihs.ac.at

${ }^{\ddagger}$ Department of Economics, University of California, Davis. Email: bcschipper@ucdavis.edu 


\section{Introduction}

In standard Bayesian games, every player is able to conceive of all players, actions, states, types, and payoff functions. Players may be uncertain only about which of the conceived types of players they actually face, i.e., which state obtains. Yet, in real life people are not just uncertain about which states obtain but some contingencies may be completely out of their mind when taking decisions. Consequently, when forming beliefs and beliefs about beliefs of other players etc., those unforeseen contingencies are left out entirely and people may not even realize that those contingencies are left out. Moreover, since several players may be involved, players may also form beliefs about the unawareness of other players, beliefs about beliefs of other players about the unawareness of yet other players etc. Standard type spaces are not adequate for capturing unawareness (Modica and Rustichini, 1994, Dekel, Lipman, and Rustichini, 1998). In this paper, we apply type spaces with unawareness, so called unawareness belief structures introduced in Heifetz, Meier, and Schipper (2013a), to develop Bayesian games with unawareness.

Heifetz, Meier, and Schipper (2013a) showed how an unawareness belief structure consisting of a lattice of spaces is adequate for modeling mutual unawareness. Every space in the lattice captures one particular collection of contingencies. Higher spaces capture richer collections of contingencies, in which states correspond to situations described by a larger set of contingencies. The join of several spaces - the lowest space at least as high as every one of them - corresponds to the union of contingencies expressible in these spaces. For every player, a type mapping associates with each state $\omega$ a probabilistic belief over states in some space that might not contain $\omega$. Conditions are imposed to relate beliefs across different spaces of the lattice. Thus, at each state, a player has a belief over all underlying uncertainties describable in the space on which this belief is concentrated. But she may be unaware regarding other uncertainties not expressible in that space. Moreover, at each state each player has beliefs about the other players' beliefs and awareness, their beliefs about other players' beliefs and awareness etc.

Unawareness belief structures capture unawareness and beliefs, beliefs about beliefs (including beliefs about unawareness), beliefs about that etc. in a parsimonious way familiar from standard type spaces. That is, hierarchies of beliefs are captured implicitly by states and type mappings. A construction of unawareness belief structures from explicit hierarchies of beliefs is complicated by the multiple awareness levels involved. In Heifetz, Meier, and Schipper (2012) we present a hierarchical construction and show the existence of a universal unawareness type space that contains all belief hierarchies. ${ }^{1}$

In this paper, we complement the unawareness belief structure with a set of actions and a utility function for each player. This defines a Bayesian game in which players may not just be uncertain about events but also unaware of some events. We also allow for uncertainty and unawareness of actions, outcomes, and players.

\footnotetext{
${ }^{1}$ Heinsalu (2013) independently proves the existence of a universal unawareness type space. However, he does not present an explicit construction of hierarchies of beliefs.
} 
The definition of a strategy in Bayesian games with unawareness is not obvious. Consider a type $\tau$ who is aware of few contingencies only, and two other types $\tau^{\prime}, \tau^{\prime \prime}$ with a richer awareness that agree with the quantitative beliefs of $\tau$ regarding the aspects of reality of which $\tau$ is aware; the beliefs of $\tau^{\prime}$ and $\tau^{\prime \prime}$ differ only concerning dimensions of the reality that $\tau$ does not conceive. Should the action taken by $\tau$ necessarily be some average of the actions taken by $\tau^{\prime}$ and $\tau^{\prime \prime}$ ? We believe that conceptually, the answer to this question is negative. When the player conceives of more parameters (e.g. motives for saving) as relevant to her decision, her optimal action (e.g. "invest in bonds" or "invest in stocks") need not be related to her optimal decision (e.g. "go shopping") when these parameters are not part of the vocabulary with which she conceives the world. ${ }^{2}$

The next step is to define Bayesian equilibrium. Analogous to standard Bayesian games, an equilibrium in a Bayesian game with unawareness is a Nash equilibrium among types. Unawareness, however, introduces a new aspect to the construction of equilibrium: A type who conceives of only few dimensions of reality does not have in mind types of other players with a wider horizon, so the optimal action of this type does not depend on the actions of these wider-horizon types. Those types, however, who assign a positive probability to this narrow-minded type, must take its action into account when optimizing. With finitely many states, existence follows from Nash (1950).

We apply Bayesian games with unawareness and Bayesian Nash equilibrium to analyze the robustness of equilibrium to small uncertainty about players' awareness of actions in strategic games. We introduce a Nash equilibrium refinement, called Unawareness Perfect Equilibrium, and prove existence in finite strategic games. For any finite strategic game we consider a sequence of Bayesian games with unawareness over actions that converge to the finite strategic game. This represents the players' uncertainty over the opponents' awareness of actions. An Unawareness Perfect Equilibrium is the limit of equilibria of this sequence as uncertainty over opponents' awareness of actions goes to zero. It turns out that our refinement characterizes undominated Nash equilibrium. At a first glance, such a characterization may look somewhat surprising because the underlying assumption of undominated Nash equilibrium is that every player's equilibrium strategy should be robust to slight mistaken choices by opponents. Every player should be cautious or prudent with respect to the rationality of opponents, which leads him to believe that no opponents' actions can be excluded from being played. This is different from our idea that there is a slight chance that due to opponents' unawareness of some actions any opponents' action may be excluded from being played. If a player is unaware of some actions, then she perceives a partial game in which these actions are missing. We assume that players are cautious or prudent in the sense of not excluding such partial games when considering the possible unawareness of opponents. An Unawareness Perfect Equilibrium strategy is robust to misperceptions or more aptly "partial perceptions" of the game by opponents.

The most prominent equilibrium concept ruling out dominated Nash equilibrium is

\footnotetext{
${ }^{2}$ This is a crucial point in which our definition of a strategy differs from the one in the parallel work of Sadzik (2006).
} 
Trembling Hand Perfect Equilibrium by Selten (1975). Every Trembling Hand Perfect Equilibrium is undominated but the converse applies to two-player games only. Selten (1975, p. 35) remarks that there is an inconsistency in the interpretation of Trembling Hand Perfect Equilibrium: "There cannot be any mistakes if the players are absolutely rational. Nevertheless, a satisfactory interpretation of equilibrium points in extensive games seems to require that the possibility of mistakes is not completely excluded." That is, Selten assumes that players are irrational with a small probability. A player is irrational if she chooses a strategy that does not maximize her payoff given her beliefs. Note that in an Unawareness Perfect Equilibrium, a player still chooses a strategy that maximizes her payoff given her beliefs but her beliefs may be constrained by her limited awareness. So, in this sense our characterization allows us to provide a justification for undominated Nash equilibrium without resorting to irrationality of players. Replacing irrational actions of opponents by uncertainty about opponents' unawareness is of conceptional significance. Behavior should be endogenous to the model but is in part (i.e., due to ad hoc trembles) exogenous in trembling hand perfect equilibrium. In contrast, unawareness and beliefs are explicitly specified as part of our game model rather than as an ad hoc assumption on behavior. The reinterpretation of undominated Nash equilibrium as equilibrium robust to "partial perceptions" of the game may be of interest to applied game theorists when contemplating which equilibrium refinement to apply in a context involving possible inattention by players.

Bayesian games with unawareness allow both for unawareness and probability zero beliefs. This raises the question about the differences between probability zero events and events that an agent is unaware of. In Appendix B, we show how to "flatten" a Bayesian game with unawareness by taking the union of all spaces and assigning zero probability to all states of which the individual is unaware. The "flattened" game is a standard Bayesian game with a standard type space; thus the Dekel-Lipman-Rustichini (1998) critique applies and unawareness becomes trivial. "Flattening" does not "change" the set of Bayesian Nash equilibria though but equilibria in the "flattened" game cannot be interpreted anymore with unawareness. The "behavioral correspondence" between Bayesian games with unawareness and the "flattened game" does not imply that unawareness has no behavioral implications (see for instance, Schipper 2013). We view our contribution precisely as defining the primitives of models in which behavior under unawareness can be studied. This may be best explained with an analogy to ambiguity. It is known that the Ellsberg paradox can be rationalized by subjective expected utility with additive probabilities on a larger state-space (see Gilboa and Schmeidler, 1994, Grabiszewski, 2013). That is, whether particular behavior is classified as ambiguity averse or consistent with subjective expected utility depends on the primitive state-space assumed by the modeler. We face a similar situation with unawareness. Interpreting behavior as behavior under unawareness and revealing unawareness requires primitives that are rich enough to model unawareness. Standard states-spaces lack the structure to identify behavior under unawareness or even talk about unawareness.

The paper is organized as follows: In the remaining subsections of the introduction, we provide a simple example illustrating the concept of unawareness perfection. In Section 
2 we outline interactive unawareness belief structures. Bayesian games with unawareness are developed in Section 3. In Section 4 we introduce the concept of unawareness perfection for strategic games, prove existence, and characterize it by undominated Nash equilibrium. We finish with a brief discussion of the related literature in Section 5. In the appendix, we extend our approach to allow for unawareness of players and discuss the relationship between standard Bayesian games and Bayesian games with unawareness. Further proofs are relegated to an appendix as well.

\subsection{A Simplified Illustration of Unawareness Perfection}

In this section we briefly discuss a simplified illustration of unawareness perfection. Although the precise construction in Section 4 is more involved, we believe that the sketched exposition put forward in this section conveys the main idea.

Consider for instance the strategic game $\gamma^{0}$ given by

\begin{tabular}{|c|c|c|}
\hline$\gamma^{0}$ & $L$ & $R$ \\
\hline$U$ & 1,1 & 2,0 \\
\hline$D$ & 0,2 & 2,2 \\
\hline
\end{tabular}

There are two pure equilibria, $(U, L)$ and $(D, R)$, and no other equilibria. Which equilibrium of game $\gamma^{0}$ is robust to player's uncertainty that the opponent may be unaware of some action?

Given the game $\gamma^{0}$, derive a partially ordered set of restricted games by considering the set of all subsets of actions for all players partially ordered by set inclusion:

\begin{tabular}{|c|c|}
\hline$\gamma^{1}$ & $L$ \\
\hline$U$ & 1,1 \\
\hline$D$ & 0,2 \\
\hline
\end{tabular}

\begin{tabular}{|c|c|}
\hline$\gamma^{2}$ & $R$ \\
\hline$U$ & 2,0 \\
\hline$D$ & 2,2 \\
\hline
\end{tabular}

\begin{tabular}{|c|c|c|}
\hline$\gamma^{3}$ & $L$ & $R$ \\
\hline$U$ & 1,1 & 2,0 \\
\hline
\end{tabular}

\begin{tabular}{|c|c|c|}
\hline$\gamma^{4}$ & $L$ & $R$ \\
\hline$D$ & 0,2 & 2,2 \\
\hline
\end{tabular}

\begin{tabular}{|c|c|}
\hline$\gamma^{5}$ & $L$ \\
\hline$U$ & 1,1 \\
\hline
\end{tabular}

\begin{tabular}{|c|c|}
\hline$\gamma^{6}$ & $L$ \\
\hline$D$ & 0,2 \\
\hline
\end{tabular}

\begin{tabular}{|c|c|}
\hline$\gamma^{7}$ & $R$ \\
\hline$U$ & 2,0 \\
\hline
\end{tabular}

\begin{tabular}{|c|c|}
\hline$\gamma^{8}$ & $R$ \\
\hline$D$ & 2,2 \\
\hline
\end{tabular}

For instance, a player in game $\gamma^{1}$ is unaware of column player's action $R$. Hence, she is unaware of games $\gamma^{0}, \gamma^{2}, \gamma^{3}, \gamma^{4}, \gamma^{7}$, and $\gamma^{8}$. However, she can envision that her opponent may be unaware of some action in $\gamma^{1}$ and may view the game to be $\gamma^{5}$ or $\gamma^{6}$. A player being unaware of action $R$ only is said to have awareness level $\gamma^{1}$.

Consider now the system of completely mixed beliefs over all restricted games including $\gamma^{0}$ in Table 1. Each row describes a completely mixed belief over games given the awareness level associated with that row. E.g., $t^{0}$ denotes the completely mixed belief over games for a player with awareness level $\gamma^{0}$. Such a player may believe with probability $t^{0}\left(\gamma^{1}\right)$ that the opponent's awareness level is $\gamma^{1}$. Such an opponent's belief about the player's awareness level is then given in turn by $t^{1}$. A player with awareness level $\gamma^{0}$ who believes with probability $t^{0}\left(\gamma^{1}\right)$ that his opponent has awareness level $\gamma^{1}$ also 
Table 1: System of Completely Mixed Beliefs

\begin{tabular}{c|cccccccccc} 
Games & $\gamma^{0}$ & $\gamma^{1}$ & $\gamma^{2}$ & $\gamma^{3}$ & $\gamma^{4}$ & $\gamma^{5}$ & $\gamma^{6}$ & $\gamma^{7}$ & $\gamma^{8}$ \\
Belief & & & & & & & & & \\
\hline$t^{0}$ & $1-\varepsilon$ & $\frac{\varepsilon}{8}$ & $\frac{\varepsilon}{8}$ & $\frac{\varepsilon}{8}$ & & $\frac{\varepsilon}{8}$ & $\frac{\varepsilon}{8}$ & $\frac{\varepsilon}{8}$ & $\frac{\varepsilon}{8}$ & $\frac{\varepsilon}{8}$ \\
$t^{1}$ & & $1-\varepsilon$ & & & & $\frac{\varepsilon}{2}$ & $\frac{\varepsilon}{2}$ & & \\
$t^{2}$ & & & $1-\varepsilon$ & & & & & $\frac{\varepsilon}{2}$ & $\frac{\varepsilon}{2}$ \\
$t^{3}$ & & & & $1-\varepsilon$ & & $\frac{\varepsilon}{2}$ & & $\frac{\varepsilon}{2}$ & \\
$t^{4}$ & & & & & $1-\varepsilon$ & & $\frac{\varepsilon}{2}$ & & $\frac{\varepsilon}{2}$ \\
$t^{5}$ & & & & & & 1 & & & \\
$t^{6}$ & & & & & & & 1 & & \\
$t^{7}$ & & & & & & & & & 1 & \\
$t^{8}$ & & & & & & & & & & \\
\end{tabular}

believes that such an opponent is unaware of $\gamma^{0}$ and believes with probability $t^{1}\left(\gamma^{5}\right)$ that the player himself has awareness level $\gamma^{5}$. Etc. Essentially this corresponds to a Bayesian game with unawareness of actions with completely mixed beliefs (that will be captured in Property (iv) in Section 4).

We are interested in finding out which equilibrium of the game $\gamma^{0}$ is robust to such beliefs about opponent's unawareness in the limit as $\varepsilon$ goes to zero, assuming that at each awareness level and for each $\varepsilon$ considered, players play Bayesian Nash equilibrium. Such equilibria we call Unawareness Perfect Equilibria. To construct such an equilibrium, consider the games at the lowest levels $\gamma^{5}, \gamma^{6}, \gamma^{7}$, and $\gamma^{8}$. Since the set of outcomes is a singleton, for any $\varepsilon$ the Bayesian equilibria are trivial in those games. Now at any higher awareness level, players must take the equilibrium behavior of unaware players in the lower games into account. Thus we can define inductively Bayesian equilibria with unawareness. When we consider games $\gamma^{8}$ to $\gamma^{1}$ in our example, this yields a unique outcome for all games except $\gamma^{2}$ and $\gamma^{4}$. In latter two games any mixtures of the row and column players, respectively, are allowed. Since $t^{0}$ is completely mixed over all lower games, every action of the opponent is assigned some strict positive weight in equilibrium at awareness level $\gamma^{0}$ as long as $\varepsilon>0$. Consequently the best reply of the type $t^{0}$ is always to play $U$ as row player and $L$ as column player. Taking $\varepsilon$ to zero selects uniquely the equilibrium $(U, L)$ as the Unawareness Perfect Equilibrium of $\gamma^{0}$. This corresponds to the undominated Nash equilibrium. In the paper, we show that this equivalence holds more generally. Every Unawareness Perfect Equilibrium of a finite strategic game is an undominated equilibrium and vice versa. Section 4 develops this in a general framework.

\section{Model}

Let $\mathcal{S}=\left\{S_{\alpha}\right\}_{\alpha \in \mathcal{A}}$ be a finite lattice of disjoint finite state-spaces, with the partial order $\succeq$ on $\mathcal{S}$. Any finite lattice is complete, i.e, each subset has a least upper bound (i.e., 
supremum) and a greatest lower bound (i.e., infimum). If $S_{\alpha}$ and $S_{\beta}$ are such that $S_{\alpha} \succeq S_{\beta}$ we say that " $S_{\alpha}$ is more expressive than $S_{\beta}$ - states of $S_{\alpha}$ describe situations with a richer vocabulary than states of $S_{\beta}{ }^{\prime \prime}{ }^{3}$. Spaces in the lattice can be more or less "rich" in terms of facts that may or may not obtain in them. The partial order relates to the "richness" of spaces. Denote by $\Omega=\bigcup_{\alpha \in \mathcal{A}} S_{\alpha}$ the disjoint union of these spaces. Each $S \in \mathcal{S}$ is assumed to be finite.

For every $S$ and $S^{\prime}$ such that $S^{\prime} \succeq S$, there is a surjective projection $r_{S}^{S^{\prime}}: S^{\prime} \longrightarrow S$, where $r_{S}^{S}$ is the identity. We interpret $r_{S}^{S^{\prime}}(\omega)$ as "the restriction of the description $\omega$ to the more limited vocabulary of $S . "$ Projections "translate" states from "more expressive" spaces to states in "less expressive" spaces by "erasing" facts that can not be expressed in a lower space. Note that the cardinality of $S$ is smaller than or equal to the cardinality of $S^{\prime}$. We require the projections to commute: If $S^{\prime \prime} \succeq S^{\prime} \succeq S$ then $r_{S}^{S^{\prime \prime}}=r_{S}^{S^{\prime}} \circ r_{S^{\prime}}^{S^{\prime \prime}}$. If $\omega \in S^{\prime}$, denote $\omega_{S}=r_{S}^{S^{\prime}}(\omega)$. If $D \subseteq S^{\prime}$, denote $D_{S}=\left\{\omega_{S}: \omega \in D\right\}$.

For $D \subseteq S$, denote $D^{\uparrow}=\bigcup_{S^{\prime} \in\left\{S^{\prime}: S^{\prime} \succeq S\right\}}\left(r_{S}^{S^{\prime}}\right)^{-1}(D)$. ("All the extensions of descriptions in $D$ to at least as expressive vocabularies.") Clearly, $D^{\uparrow}$ is a subset of $\Omega$.

An event is a pair $(E, S)$, where $E=D^{\uparrow}$ with $D \subseteq S$, where $S \in \mathcal{S}$. $D$ is called the base and $S$ the base-space of $(E, S)$, denoted by $S(E)$. If $E \neq \emptyset$, then $S$ is uniquely determined by $E$ and, abusing notation, we write $E$ for $(E, S)$. Otherwise, we write $\emptyset^{S}$ for $(\emptyset, S)$. Note that not every subset of $\Omega$ represents an event. Some fact may obtain in a subset of a space. Then this fact should be also "expressible" in "more expressive" spaces. Therefore the event contains not only the particular subset but also its inverse images in "more expressive" spaces.

Let $\Sigma$ be the set of events of $\Omega$, i.e., $D^{\uparrow}$ such that $D \in 2^{S}$, for some state-space $S \in \mathcal{S}$. See Heifetz, Meier, and Schipper (2006, 2008, 2013a) for further details on the event structure.

Let $\Delta(S)$ be the set of probability measures on $S$. We consider this set itself as a measurable space endowed with the $\sigma$-field $\mathcal{F}_{\Delta(S)}$ generated by the sets $\{\mu \in \Delta(S): \mu(D) \geq p\}$, where $D \in 2^{S}$ and $p \in[0,1]$.

For a probability measure $\mu \in \Delta\left(S^{\prime}\right)$, the marginal $\mu_{\mid S}$ of $\mu$ on $S \preceq S^{\prime}$ is defined by

$$
\mu_{\mid S}(D):=\mu\left(\left(r_{S}^{S^{\prime}}\right)^{-1}(D)\right), \quad D \in 2^{S}
$$

Let $S_{\mu}$ be the space on which $\mu$ is a probability measure. Whenever for some event $E$ we have $S_{\mu} \succeq S(E)$ then we abuse notation slightly and write

$$
\mu(E)=\mu\left(E \cap S_{\mu}\right)
$$

If $S(E) \npreceq S_{\mu}$, then we say that $\mu(E)$ is undefined.

\footnotetext{
${ }^{3}$ Here and in what follows, phrases within quotation marks hint at intended interpretations, but we emphasize that these interpretations are not part of the definition of the set-theoretic structure.
} 
$I$ is the nonempty finite set of players. For every player, each state gives rise to a probabilistic belief over states in some space.

Definition 1 For each player $i \in I$ there is a type mapping $t_{i}: \Omega \longrightarrow \bigcup_{\alpha \in \mathcal{A}} \Delta\left(S_{\alpha}\right)$. We require the type mapping $t_{i}$ to satisfy the following properties: ${ }^{4}$

(i) Confinement: If $\omega \in S^{\prime}$ then $t_{i}(\omega) \in \triangle(S)$ for some $S \preceq S^{\prime}$.

(ii) If $S^{\prime \prime} \succeq S^{\prime} \succeq S, \omega \in S^{\prime \prime}$, and $t_{i}(\omega) \in \triangle\left(S^{\prime}\right)$ then $t_{i}\left(\omega_{S}\right)=t_{i}(\omega)_{\mid S}$.

(iii) If $S^{\prime \prime} \succeq S^{\prime} \succeq S, \omega \in S^{\prime \prime}$, and $t_{i}\left(\omega_{S^{\prime}}\right) \in \triangle(S)$ then $S_{t_{i}(\omega)} \succeq S$.

(iv) Introspection: $t_{i}(\omega)\left(\left\{\omega^{\prime} \in \Omega: t_{i}\left(\omega^{\prime}\right)_{\mid S_{t_{i}(\omega)}}=t_{i}(\omega)\right\}\right)=1$.

$t_{i}(\omega)$ represents player $i$ 's belief at state $\omega$. Properties (i) to (iii) guarantee the consistent fit of beliefs and awareness at different state-spaces. Confinement means that at any given state $\omega \in \Omega$ an player's belief is concentrated on states that are all described with the same "vocabulary" - the "vocabulary" available to the player at $\omega$. This "vocabulary" may be less expressive than the "vocabulary" used to describe statements in the state $\omega . "$

Properties (ii) to (iii) compare the types of an player in a state $\omega \in S^{\prime}$ and its projection to $\omega_{S}$, for some $S \preceq S^{\prime}$. Consider property (ii). Suppose a player's awareness level at $\omega$ is $S^{\prime}$. What should the player's beliefs be at a poorer description of $\omega$ at an awareness level $S$ below $S^{\prime}$ ? Property (ii) says that the player should hold the same belief over an event $E$ as he does at $\omega$ provided that he is still aware of the event $E$. In this sense, the types at $\omega$ and $\omega_{S}$ just differ in their awareness. Property (iii) means that at $\omega$ a player cannot be unaware of an event that she is aware of at the projected state $\omega_{S^{\prime}}$.

Property (iv) means that at every state, player $i$ is certain about her own beliefs. More precisely, for every state $\omega$, the type of player $i$ at $\omega$ is certain of the set of states at which player $i$ 's type or the marginal thereof coincides with her type at $\omega$. This property implies introspection (i.e., Property (va) in Proposition 4 in Heifetz, Meier, and Schipper, 2013a).

When $t_{i}(\omega) \in \Delta(S)$, then we often refer to $S$ as the awareness level of type $t_{i}(\omega)$.

Definition 2 We denote by $\underline{\mathcal{S}}:=\left\langle\mathcal{S},\left(r_{S_{\beta}}^{S_{\alpha}}\right)_{S_{\beta} \preceq S_{\alpha}},\left(t_{i}\right)_{i \in I}\right\rangle$ a finite interactive unawareness belief structure.

Apart from restricting ourselves to a finite lattice of finite spaces, the model outlined in this section corresponds to unawareness belief structures introduced in Heifetz, Meier, and Schipper (2013a). Heifetz, Meier, and Schipper (2012) provide an explicit hierarchical construction and show the existence of a universal unawareness type space.

\footnotetext{
${ }^{4}$ Recall that $S_{\mu}$ is the space on which $\mu$ is a probability measure. Thus, $S_{t_{i}(\omega)}$ is the space on which $t_{i}(\omega)$ is a probability measure.
} 


\section{Bayesian Games with Unawareness}

In this section, we generalize strategic games with incomplete information à la Harsanyi $(1967 / 68)$ and Mertens and Zamir (1985, Section 5) to include also unawareness. For convenience, we restrict ourselves to finite games. Moreover, we focus in this section on unawareness of payoff-relevant events and unawareness of actions; unawareness of players is relegated to Appendix A.

Unawareness of payoff-relevant events is rather straight-forward to model. We simply replace the type-space of a standard Bayesian game with an unawareness structure. To model properly also unawareness of actions, we need to work a little harder. In standard Bayesian game theory, ignorance of actions is modeled by the assumption that players will never use such actions, because extremely low payoffs (i.e., highly negative) are assigned to those actions (see the discussion in Harsanyi, 1967, p. 168). We do not follow this convention here. Even in standard Bayesian games this convention is questionable, because it applies to rational players only. If there is lack of common belief of rationality then a player's type being ignorant of an action is indeed different from her obtaining a very low payoff from playing this action (see $\mathrm{Hu}$ and Stuart, 2001, for a discussion).

Denote $\left[t_{i}(\omega)\right]:=\left\{\omega^{\prime} \in \Omega: t_{i}\left(\omega^{\prime}\right)=t_{i}(\omega)\right\}$. This is the set of states at which player $i$ has the same type as in state $\omega$.

Definition 3 A Bayesian game with unawareness

$$
\Gamma(\underline{\mathcal{S}})=\left\langle\mathcal{S},\left(r_{S_{\beta}}^{S_{\alpha}}\right)_{S_{\beta} \preceq S_{\alpha}},\left(t_{i}\right)_{i \in I},\left(M_{i}\right)_{i \in I},\left(\mathcal{M}_{i}\right)_{i \in I},\left(u_{i}\right)_{i \in I}\right\rangle
$$

consists of a unawareness belief structure $\underline{\mathcal{S}}=\left\langle\mathcal{S},\left(r_{S_{\beta}}^{S_{\alpha}}\right)_{S_{\beta} \preceq S_{\alpha}},\left(t_{i}\right)_{i \in I}\right\rangle$ and

(i) a nonempty finite set of actions $M_{i}$, for $i \in I$, and a correspondence $\mathcal{M}_{i}: \Omega \longrightarrow$ $2^{M_{i}} \backslash\{\emptyset\}$, for $i \in I$, such that for any nonempty subset of actions $M_{i}^{\prime} \subseteq M_{i}$, $\left[M_{i}^{\prime}\right]:=\left\{\omega \in \Omega: M_{i}^{\prime} \subseteq \mathcal{M}_{i}(\omega)\right\}$ is an event (in the unawareness belief structure), and $\omega^{\prime}, \omega^{\prime \prime} \in\left[t_{i}(\omega)\right] \cap S_{t_{i}(\omega)}$ implies $\mathcal{M}_{i}\left(\omega^{\prime}\right)=\mathcal{M}_{i}\left(\omega^{\prime \prime}\right)$, for all $\omega \in \Omega$,

(ii) for every $i \in I$, a utility function $u_{i}: \bigcup_{\omega \in \Omega}\left(\left(\prod_{j \in I} \mathcal{M}_{j}(\omega)\right) \times\{\omega\}\right) \longrightarrow \mathbb{R}$.

The interpretation is as follows: At the beginning of a game, a state $\omega \in \Omega$ is realized. Player $i$ does not observe the state but receives a signal $t_{i}(\omega)$ that provides her with some information about the state or projections thereof to lower spaces. I.e., if $\omega$ obtains, player $i$ is of type $t_{i}(\omega)$. This signal is a belief about the likelihood of events on a certain space including payoff-relevant events. A player's utility depends on her action, the actions chosen by other players as well as the state. Since players may be uncertain about the state $\omega$, we assume that the player's preference is represented by the expected value of 
the utility that depends on action-profiles of players and states, where the expectation is taken with respect to the player $i$ 's type $t_{i}(\omega)$ and the types' mixed strategies.

Which actions a player $i$ has available at what state is described explicitly by the correspondence $\mathcal{M}_{i}$. Any set of available actions is associated with an event in our unawareness belief structure. We require that each type of each player has a well-defined set of actions. That is, we exclude that at a state, a player considers it possible that she has an action available, which, in fact, is not available to her in this state. This is to avoid the conceptional problem of defining what should happen if a player is to take an action that is not available to her. At the same time, we allow in addition to unawareness of other players' actions also for unawareness of a player's own actions, because it is a realistic feature. This means formally that the sets of available actions should be constant on a player's type $\left[t_{i}(\omega)\right]$ restricted to the set of states that this type is aware of, that is, the set of states $S_{t_{i}(\omega)}$. Without the restriction to $S_{t_{i}(\omega)}$, a player's type could not be unaware of own actions. If $\omega \notin S_{t_{i}(\omega)}$, then it is possible that $\mathcal{M}_{i}\left(\omega^{\prime}\right)$ is a proper subset of $\mathcal{M}_{i}(\omega)$, for $\omega^{\prime} \in\left[t_{i}(\omega)\right] \cap S_{t_{i}(\omega)}$. Finally, observe that since outcomes consist of profiles of actions, players in a Bayesian game with unawareness of actions may also be unaware of outcomes in the game.

Note that we allow unawareness to affect payoffs in an arbitrary way. One may consider letting $u_{i}(\cdot, \omega)$ be a convex combination of all $u_{i}\left(\cdot, \omega^{\prime}\right), \omega^{\prime} \in\left(r_{S}^{S^{\prime}}\right)^{-1}(\omega)$, for $\omega \in S$ and $S^{\prime} \succeq S$. While this may be natural in some applications such as the speculation example in Heifetz, Meier, and Schipper (2013a, Section 1.1), it would preclude situations in which the mere awareness of an event may reduce expected payoffs.

Let $\Delta\left(M_{i}\right)$ be the set of mixed actions for player $i \in I$, that is, the set of probability distributions on the finite set $M_{i}$.

Definition 4 A strategy of player $i$ in a Bayesian game with unawareness is a function $\sigma_{i}: \Omega \longrightarrow \Delta\left(M_{i}\right)$ such that for all $\omega \in \Omega$,

(i) $\sigma_{i}(\omega) \in \Delta\left(\mathcal{M}_{i}\left(\omega_{S_{t_{i}(\omega)}}\right)\right)$, and

(ii) $t_{i}\left(\omega^{\prime}\right)=t_{i}(\omega)$ implies $\sigma_{i}\left(\omega^{\prime}\right)=\sigma_{i}(\omega)$.

A strategy specifies for each player and state a probability distribution over her perceived set of actions. In standard Bayesian games without unawareness, one interpretation of a strategy assumes an ex-ante point of view of the player before she knows her type. This interpretation is misleading in a game with unawareness, since if a player is aware of all her types ex-ante she should be also aware of all types interim, i.e., after learning her type (and her awareness). Hence, in the case of unawareness, the ex-ante notion of strategy is a construct of the game theorist rather than an object of choice for a player.

In Bayesian games with unawareness we subscribe to a second interpretation of Bayesian strategy from an interim point of view: Given a player $i$ and type $t_{i}(\omega)$, she has 
an "awareness level" $S_{t_{i}(\omega)} \in \mathcal{S}$. That is, she can consider strategies of her opponents in $l\left(S_{t_{i}(\omega)}\right)$, where $l(S):=\left\{S^{\prime} \in \mathcal{S}: S^{\prime} \preceq S\right\}$ is the complete sublattice of $\mathcal{S}$ with $S$ being the upmost space. This interpretation is sound precisely because of Proposition 2 and Remark 1 below: To best-respond to the strategies of the other player-types, a type of a player needs only to reason about the strategies of player-types that she is aware of. Only strategies of these player-types enter in her utility maximization problem.

At this point, it may be useful to illustrate the definitions with an example due to Feinberg (2005). It allows us also compare our approach with his work.

Example 1 (Feinberg, 2005) Consider the strategic $3 \times 3$ game

\begin{tabular}{c|c|c|c|c|}
\multicolumn{5}{c}{ Colin } \\
\cline { 2 - 5 } Rowena & $b_{1}$ & $b_{2}$ & $b_{3}$ \\
\cline { 2 - 5 } & $a_{1}$ & 0,2 & 3,3 & 0,2 \\
\cline { 2 - 5 } & $a_{2}$ & 2,2 & 2,1 & 2,1 \\
\cline { 2 - 5 }$a_{3}$ & 1,0 & 4,0 & 0,1 \\
\hline
\end{tabular}

This game has a unique dominance solvable Nash equilibrium, $\left(a_{2}, b_{1}\right)$. Consider now a game with unawareness: The set of players remains unchanged, Rowena, $R$, and Colin, $C$. There are two state-spaces, $S$ and $S^{\prime}$ with $S \succ S^{\prime}$. In particular, $S=\left\{\omega_{1}, \omega_{2}\right\}$ and $S^{\prime}=\left\{\omega_{3}\right\}$. The information structure is given by the type mappings

$$
\begin{aligned}
& t_{R}\left(\omega_{1}\right)\left(\left\{w_{2}\right\}\right)=t_{R}\left(\omega_{2}\right)\left(\left\{w_{2}\right\}\right)=t_{R}\left(\omega_{3}\right)\left(\left\{\omega_{3}\right\}\right)=1, \\
& t_{C}\left(\omega_{1}\right)\left(\left\{\omega_{1}\right\}\right)=t_{C}\left(\omega_{2}\right)\left(\left\{\omega_{3}\right\}\right)=t_{C}\left(\omega_{3}\right)\left(\left\{\omega_{3}\right\}\right)=1 .
\end{aligned}
$$

Actions are specified by

$$
\begin{gathered}
\mathcal{M}_{R}\left(\omega_{1}\right)=\mathcal{M}_{R}\left(\omega_{2}\right)=\left\{a_{1}, a_{2}, a_{3}\right\}, \mathcal{M}_{R}\left(\omega_{3}\right)=\left\{a_{1}, a_{2}\right\} \\
\mathcal{M}_{C}\left(\omega_{1}\right)=\mathcal{M}_{C}\left(\omega_{2}\right)=\mathcal{M}_{C}\left(\omega_{3}\right)=\left\{b_{1}, b_{2}, b_{3}\right\}
\end{gathered}
$$

The information structure is depicted in Figure 1. The solid arrows and ellipses represent Rowena's information structure, while Colin's information structure is depicted with intermitted arrows and ellipses. At states $\omega_{1}$ and $\omega_{2}$, payoffs are given by the above payoff matrix. At state $\omega_{3}$, payoffs are given by the sub-matrix spanned by rows $a_{1}$ and $a_{2}$ and columns $b_{1}, b_{2}$, and $b_{3}$ in the above matrix, i.e.,

\begin{tabular}{c|c|c|c|c|}
\multicolumn{4}{c}{ Colin } \\
\cline { 2 - 5 } Rowena & $b_{1}$ & $b_{2}$ & $b_{3}$ \\
\cline { 2 - 5 } & $a_{1}$ & 0,2 & 3,3 & 0,2 \\
\cline { 2 - 5 } & $a_{2}$ & 2,2 & 2,1 & 2,1 \\
\cline { 2 - 5 } & &
\end{tabular}

We claim that

$$
\left(\sigma_{R}(\omega), \sigma_{C}(\omega)\right)=\left\{\begin{array}{lll}
\left(a_{3}, b_{3}\right) & \text { if } & \omega=\omega_{1} \\
\left(a_{3}, b_{2}\right) & \text { if } & \omega=\omega_{2} \\
\left(a_{1}, b_{2}\right) & \text { if } & \omega=\omega_{3}
\end{array}\right.
$$


Figure 1: Information Structure in Example 1

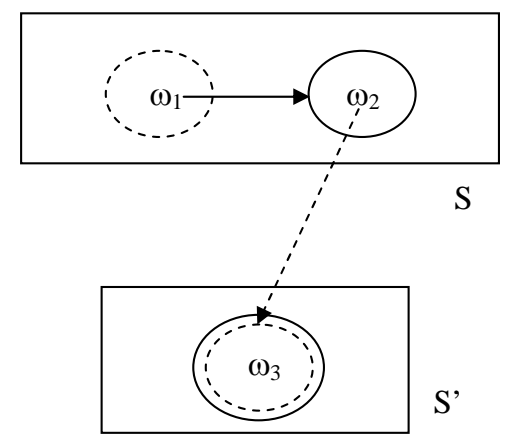

is an equilibrium. To see this, note that the game at $\omega_{3}$ has two pure equilibria, $\left(a_{2}, b_{1}\right)$ and $\left(a_{1}, b_{2}\right)$ in the $S^{\prime}$-partial game, where the latter is payoff dominant. At $\omega_{3}$, both players are unaware of action $a_{3}$ in $\omega_{3}$. The unique dominance solvable Nash equilibrium $\left(a_{2}, b_{1}\right)$ of the original game (without unawareness of actions) remains an equilibrium because none of the players is unaware of an equilibrium action and equilibrium actions remain best responses if some other actions are deleted. Moreover, after deleting action $a_{3}$ (the action both players are unaware of at state $\omega_{3}$ in $S^{\prime}$ ), the game has another Nash equilibrium $\left(a_{1}, b_{2}\right)$. At $\omega_{1}$, both players are aware of all actions but Rowena believes that Colin is unaware of action $a_{3}$. Hence Rowena believes that Colin thinks that $\left(a_{1}, b_{2}\right)$ is a Nash equilibrium. Rowena's best response to Colin playing $b_{2}$ is $a_{3}$. Moreover, since at $\omega_{1}$ Colin is aware of all actions and he believes that Rowena believes that Colin is unaware of action $a_{3}$, his best response to Rowena playing $a_{3}$ is $b_{3}$. Note that in this equilibrium at $\omega_{1}$, both receive a low payoff (compared to the Nash equilibria discussed previously).

Feinberg (2005) obtains $\left(a_{3}, b_{3}\right)$ as an equilibrium if both players are aware of all actions, Rowena is 'unaware' that Colin is aware of all of her actions, and Colin is 'aware' that Rowena is 'aware' of Colin being unaware of $a_{3} .{ }^{5}$ That is, in Feinberg (2005) a player can be aware of an event but unaware that somebody else is aware of it. This is in contrast to our unawareness belief structure, where according to Proposition 8, 1., in Heifetz, Meier, and Schipper (2008) a player is aware of an event if and only if she is aware that somebody else could be aware of it. That is, if a player can reason about some issue then she can also reason that somebody else can reason about that issue. We obtain $\left(a_{3}, b_{3}\right)$ as an equilibrium if both players are aware of all actions, Rowena does not believe that Colin is aware of $a_{3}$, and Colin believes that Rowena believes that Colin is unaware of $a_{3}$. The example suggests, that higher order 'awareness' in Feinberg (2005) operates like belief in our unawareness belief structure. Note however, that Feinberg (2005) does not define a notion of belief in his framework.

\footnotetext{
${ }^{5}$ When writing '...', we indicate that those notions differ from our notions used in this paper.
} 
Like in standard Bayesian games, an equilibrium of a Bayesian game with unawareness is a Nash equilibrium of a strategic game in which types of players are the "players". The actions available to the type of player $i$ at state $\omega$ are the actions of player $i$. The utility function of the type of player $i$ at $\omega$ is the expected utility function, given player $i$ 's awareness and belief over states at $\omega$. In an equilibrium of a Bayesian game with unawareness, the type of every player chooses an optimal mixture of actions she is aware of, given her awareness, belief and the choices of the types of the other players.

Denote $\sigma_{S_{t_{i}(\omega)}}:=\left(\left(\sigma_{j}\left(\omega^{\prime}\right)\right)_{j \in I}\right)_{\omega^{\prime} \in S_{t_{i}(\omega)}}$. The expected utility of player-type $\left(i, t_{i}(\omega)\right)$ from the strategy profile $\sigma_{S_{t_{i}(\omega)}}$ is given by

$$
\begin{aligned}
& U_{\left(i, t_{i}(\omega)\right)}\left(\sigma_{S_{t_{i}(\omega)}}\right):= \\
& \sum_{\omega^{\prime} \in S_{t_{i}(\omega)}} \sum_{m \in \prod_{j \in I} \mathcal{M}_{j}\left(\omega_{S_{t_{j}\left(\omega^{\prime}\right)}^{\prime}}^{\prime}\right)}\left(\prod_{j \in I} \sigma_{j}\left(\omega^{\prime}\right)\left(\left\{m_{j}\right\}\right)\right) \cdot u_{i}\left(\left(m_{j}\right)_{j \in I}, \omega^{\prime}\right) t_{i}(\omega)\left(\left\{\omega^{\prime}\right\}\right) .
\end{aligned}
$$

Definition 5 (Equilibrium) Given a Bayesian game with unawareness $\Gamma(\underline{\mathcal{S}})$, define the associated strategic game by

(i) $\left\{\left(i, t_{i}(\omega)\right): \omega \in \Omega\right.$ and $\left.i \in I\right\}$ is the set of players,

and for each player $\left(i, t_{i}(\omega)\right)$,

(ii) the set of mixed strategies is $\Delta\left(\mathcal{M}_{i}\left(\omega_{S_{t_{i}}(\omega)}\right)\right.$, and

(iii) the utility function is given by Equation (1).

A profile $\left(\sigma_{i}\right)_{i \in I}$ is an equilibrium of the Bayesian game with unawareness if and only if the following is an equilibrium of the associated strategic game: $\left(i, t_{i}(\omega)\right)$ plays $\sigma_{i}(\omega)$, for all $i \in I$ and $\omega \in \Omega$.

Existence of equilibrium follows now directly from Nash's (1950) theorem.

Proposition 1 (Existence) Every finite Bayesian game with unawareness has an equilibrium. bound.

Recall $l(S):=\left\{S^{\prime} \in \mathcal{S}: S^{\prime} \preceq S\right\} . l(S)$ is a sublattice with $S$ as the least upper

Definition 6 Given a Bayesian game with unawareness

$$
\Gamma(\underline{\mathcal{S}})=\left\langle\mathcal{S},\left(r_{S_{\beta}}^{S_{\alpha}}\right)_{S_{\beta} \preceq S_{\alpha}},\left(t_{i}\right)_{i \in I},\left(M_{i}\right)_{i \in I},\left(\mathcal{M}_{i}\right)_{i \in I},\left(u_{i}\right)_{i \in I}\right\rangle,
$$


we can define for any $S^{\prime} \in \mathcal{S}$ an $S^{\prime}$-partial Bayesian game with unawareness

$$
\Gamma \underline{\left(l\left(S^{\prime}\right)\right)}=\left\langle l\left(S^{\prime}\right),\left(r_{S_{\beta}}^{S_{\alpha}}\right)_{S_{\beta} \preceq S_{\alpha} \preceq S^{\prime}},\left(t_{i}\right)_{i \in I},\left(M_{i}\right)_{i \in I},\left(\mathcal{M}_{i}^{\prime}\right)_{i \in I},\left(u_{i}\right)_{i \in I}\right\rangle,
$$

where for any $i \in I, \mathcal{M}_{i}^{\prime}$ is $\mathcal{M}_{i}$ restricted to $\Omega^{\prime}=\bigcup_{S^{\prime \prime} \in l\left(S^{\prime}\right)} S^{\prime \prime}$.

Note that contrary to an ordinary Bayesian game, the game is not "common knowledge" among the players. Let $\Gamma(\underline{\mathcal{S}})=\left\langle\mathcal{S},\left(r_{S_{\beta}}^{S_{\alpha}}\right)_{S_{\beta} \preceq S_{\alpha}},\left(t_{i}\right)_{i \in I},\left(M_{i}\right)_{i \in I},\left(\mathcal{M}_{i}\right)_{i \in I},\left(u_{i}\right)_{i \in I}\right\rangle$ be a Bayesian game with unawareness. At $\omega \in \Omega$, the game conceived by player $j$ is $\Gamma \underline{\left.\Gamma\left(S_{t_{j}(\omega)}\right)\right)}=\left\langle l\left(S_{t_{j}(\omega)}\right),\left(r_{S_{\beta}}^{S_{\alpha}}\right)_{S_{\beta} \preceq S_{\alpha} \preceq S_{t_{j}(\omega)}},\left(t_{i}\right)_{i \in I},\left(M_{i}\right)_{i \in I},\left(\mathcal{M}_{i}^{\prime}\right)_{i \in I},\left(u_{i}\right)_{i \in I}\right\rangle$, where the lattice of spaces is replaced by the sublattice $l\left(S_{t_{j}(\omega)}\right)$ with $S_{t_{j}(\omega)}$ as the upmost space, and the domains of $t_{i}$ and $u_{i}$ are restricted to $\Omega^{\prime}=\bigcup_{S \in l\left(S_{t_{j}(\omega)}\right)} S$. Type $t_{j}(\omega)$ of player $j$ can conceive of all events expressible in spaces of the sublattice $l\left(S_{t_{j}(\omega)}\right)$.

The following proposition shows that we can naturally extend equilibria from "lower to higher awareness levels" by taking the equilibrium strategies at the "lower awareness levels" to be fixed and looking for a fixed point at "higher awareness levels". For a proof, see the proof of the more general Proposition 4 in Appendix A.

Proposition 2 ("Upwards Induction") Given a Bayesian game with unawareness $\left\langle\mathcal{S},\left(r_{S_{\beta}}^{S_{\alpha}}\right)_{S_{\beta} \preceq S_{\alpha}},\left(t_{i}\right)_{i \in I},\left(M_{i}\right)_{i \in I},\left(\mathcal{M}_{i}\right)_{i \in I},\left(u_{i}\right)_{i \in I}\right\rangle$, consider for $S^{\prime}, S^{\prime \prime} \in \mathcal{S}$ with $S^{\prime} \preceq$ $S^{\prime \prime}$ the $S^{\prime}$-partial (resp. $S^{\prime \prime}$-partial) Bayesian game with unawareness. If $I, \Omega$, and $\left(M_{i}\right)_{i \in I}$ are finite, then for every equilibrium of the $S^{\prime}$-partial Bayesian game, there is an equilibrium of the $S^{\prime \prime}$-partial Bayesian game in which equilibrium strategies of playertypes in $\left\{\left(i, t_{i}(\omega)\right): \omega \in \Omega^{\prime}=\bigcup_{S \in l\left(S^{\prime}\right)} S\right.$ and $\left.i \in I\right\}$ are identical with the equilibrium strategies in the $S^{\prime}$-partial Bayesian game.

This proposition suggests a procedure for constructing equilibria in Bayesian games with unawareness. We start with an equilibrium in each $\hat{S}$-partial Bayesian game with unawareness, for the lowest space $\hat{S}$ of the lattice, and extend it step-by-step to higher spaces by finding a fixed-point taking the strategies of player-types in the lower spaces as given.

For some strategic situations, Proposition 2 suggests that players who are unaware may have commitment power (although they do not understand that they are committed) compared to players with a "higher awareness level". This is so because types with "lower awareness levels" do not react to types of which they are unaware. Types with "higher awareness" must take strategies of types with "lower awareness" as given. It is easy to construct examples of Bayesian games with unawareness in which the "value of awareness" may be negative. 
We can also restrict an equilibrium from higher awareness levels to lower awareness. This is so, because if player-types play an equilibrium in a game that allows for "higher awareness levels", then those player-types still play optimally at "lower awareness levels". This is stated more formally in Remark 1, which follows from the consistency of Nash equilibrium (see Peleg and Tijs, 1996, and Peleg, Potters, and Tijs, 1996) ${ }^{6}$ :

Remark 1 Let $\left\langle\mathcal{S},\left(r_{S_{\beta}}^{S_{\alpha}}\right)_{S_{\beta} \preceq S_{\alpha}},\left(t_{i}\right)_{i \in I},\left(M_{i}\right)_{i \in I},\left(\mathcal{M}_{i}\right)_{i \in I},\left(u_{i}\right)_{i \in I}\right\rangle$ be a Bayesian game with unawareness. Consider for $S^{\prime}, S^{\prime \prime} \in \mathcal{S}$ with $S^{\prime} \preceq S^{\prime \prime}$ the $S^{\prime}$-partial (resp. $S^{\prime \prime}$ partial) Bayesian game with unawareness. Then for every equilibrium of the $S^{\prime \prime}$-partial Bayesian game there is a unique equilibrium of the $S^{\prime}$-partial Bayesian game in which the equilibrium strategies of player-types in $\left\{\left(i, t_{i}(\omega)\right): \omega \in \Omega^{\prime}=\bigcup_{S \in l\left(S^{\prime}\right)} S\right.$ and $\left.i \in I\right\}$ are identical to the equilibrium strategies of the $S^{\prime \prime}$-partial Bayesian game.

\section{Unawareness Perfection}

In this section, we apply our framework to analyze Nash equilibria of strategic games that are robust to small uncertainty about awareness of actions. The main idea is to associate with a strategic game a sequence of Bayesian games with unawareness of actions and then consider the limit of equilibria of these games as uncertainty over awareness of opponents' actions vanishes and players become certain that everybody is aware of all actions.

We start by defining a sequence of specific Bayesian games with unawareness of actions. Let $\tilde{\gamma}=\left\langle I,\left(\tilde{M}_{i}\right)_{i \in I},\left(\tilde{v}_{i}\right)_{i \in I}\right\rangle$ be a finite strategic game with a finite set of players $I=\{1, \ldots, n\}$, for each player $i \in I$ a finite nonempty set of actions $\tilde{M}_{i}$ and a payoff function $\tilde{v}_{i}: \tilde{M} \longrightarrow \mathbb{R}$, where $\tilde{M}:=\prod_{i \in I} \tilde{M}_{i}$.

Given such a strategic game, we append for each player $i \in I$ a "default" action $d_{i}$ and extend the payoff functions such that any player's default action is strictly dominated by every of her other actions. Moreover, whenever some player $i \in I$ takes her default action, then all other players' payoffs can be arbitrary. We interpret this default action as "do nothing". Intuitively, a player should always have some action available even if this action just amounts to "do nothing". The default action is a technical device that allows us to obtain a unique "lowest" game in the set of games defined below.

More formally, for all $i \in I$, let $M_{i}=\tilde{M}_{i} \cup\left\{d_{i}\right\}$. For all $i \in I$, define $v_{i}: M \longrightarrow \mathbb{R}$ with $v_{i}(m)=\tilde{v}_{i}(m)$ for all $m \in \tilde{M}$ and $v_{i}\left(d_{i}, m_{-i}\right)<v_{i}\left(m_{i}, m_{-i}\right)$ for all $m_{i} \in \tilde{M}_{i}$ and all $m_{-i} \in M_{-i}=\prod_{j \in I \backslash\{i\}} M_{j}$. For all $m_{-i}=\left(m_{1}, \ldots, m_{i-1}, m_{i+1}, \ldots, m_{n}\right) \in M_{-i}$ with $m_{j}=d_{j}$ for some $j \in I \backslash\{i\}, v_{i}\left(m_{i}, m_{-i}\right)$ can be arbitrary for any $m_{i} \in \tilde{M}_{i}$. We write $\gamma=\left\langle I,\left(M_{i}\right)_{i \in I},\left(v_{i}\right)_{i \in I}\right\rangle$ for the strategic game with default actions.

We call a subset $L^{\prime}$ of a lattice $L$ a meet-sublattice if it is a lattice with respect to the order induced by $L$ and the meet of any two elements of $L^{\prime}$ is the meet of the two

\footnotetext{
${ }^{6}$ We thank an anonymous reviewer for drawing our attention to these references.
} 
elements in the lattice $L$. (Note that we do not require the join of any two elements of $L^{\prime}$ to be the join in $L$.)

Given the strategic game $\gamma$, we define a set of restricted games as follows: For any nonempty $M_{i}^{\prime}$ with $M_{i}^{\prime} \subseteq M_{i}$ and $d_{i} \in M_{i}^{\prime}, M^{\prime}:=\prod_{i \in I} M_{i}^{\prime}$, define a restricted strategic game $\left\langle I,\left(M_{i}^{\prime}\right)_{i \in I},\left(\left.v_{i}\right|_{M^{\prime}}\right)_{i \in I}\right\rangle$ where $\left.v_{i}\right|_{M^{\prime}}$ is player $i$ 's payoff function in $\gamma$ restricted to outcomes in $M^{\prime}$. Note that for each player the action set of a restricted game contains her default action. Let $\perp$ denote the trivial game in which each player just has her default action. A set of restricted games derived from $\gamma$ is rich, if for all $\left(m_{i}\right)_{i \in I} \in \tilde{M}$ the restricted games $\left\langle I,\left(\left\{m_{i}, d_{i}\right\}\right)_{i \in I},\left(\left.v_{i}\right|_{\prod_{j \in I}\left\{m_{j}, d_{j}\right\}}\right)_{i \in I}\right\rangle, \gamma$, and $\perp$ belong to this set of restricted games. A partial order on the set of all restricted games is defined by set inclusion of $\prod_{i \in I} M_{i}$. Note that the set of all restricted games is a finite lattice. The meet is defined by the intersection of action sets. Let $G(\gamma)$ denote a rich meet-sublattice of restricted games derived from $\gamma$, and let $\preceq$ denote the partial order on $G(\gamma) .^{7}$ If $\alpha \in G(\gamma)$, we denote by $G(\alpha)$ the sublattice of strategic games $\beta \in G(\gamma)$ for which $\beta \preceq \alpha$.

For notational convenience, define $I^{0}:=I \cup\{0\}$. For each $\alpha \in G(\gamma)$, we define a space of states

$$
S_{\alpha}=\left\{\omega: \omega=\left(\alpha_{i}\right)_{i \in I^{0}} \text { with } \alpha_{0}=\alpha \text { and } \alpha_{i} \in G(\alpha) \text { for every } i \in I\right\}
$$

Intuitively, a state in $S_{\alpha}$ shall describe which game each player perceives together with the index $\alpha_{0}=\alpha$ for the state space $S_{\alpha}$. In a state in $S_{\alpha}$ no player can perceive a game $\beta \in G(\gamma)$ that is "more expressive than or incomparable to" $\alpha$. Let $\mathcal{S}_{\gamma}=\left\{S_{\alpha}\right\}_{\alpha \in G(\gamma)}$. By definition (by the first component of the states), these spaces are disjoint. The set $\mathcal{S}_{\gamma}$ is a lattice of disjoint state-spaces, where the partial order is defined by extending $\preceq$ to $\mathcal{S}_{\gamma}$ by $S_{\alpha} \succeq S_{\beta}, S_{\alpha}, S_{\beta} \in \mathcal{S}_{\gamma}$ if and only if $\alpha \succeq \beta, \alpha, \beta \in G(\gamma)$. As before, the union of all spaces is denoted by $\Omega$.

For all $\alpha, \beta \in G(\gamma)$ with $\alpha \succeq \beta$, the projections $r_{\beta}^{\alpha}: S_{\alpha} \longrightarrow S_{\beta}$ are defined by the following rule: If $\omega=\left(\alpha_{i}\right)_{i \in I^{0}} \in S_{\alpha}$, then $r_{\beta}^{\alpha}(\omega)=\left(\beta_{i}\right)_{i \in I^{0}}$ with $\beta_{i}=\inf \left\{\alpha_{i}, \beta\right\}$ for all $i \in I^{0}$. The inf does always exist since $G(\gamma)$ is a lattice. The proof of the following remark is contained in Appendix C.

Remark 2 For all $\alpha \in G(\gamma), r_{\alpha}^{\alpha}=i d_{S_{\alpha}}$. Projections commute, i.e., for any $\alpha, \beta, \delta \in$ $G(\gamma)$ with $\alpha \succeq \beta \succeq \delta, r_{\delta}^{\alpha}=r_{\delta}^{\beta} \circ r_{\beta}^{\alpha}$.

Next, we define for each player $i \in I$ a sequence of type mappings $t_{i}^{k}: \Omega \longrightarrow$ $\bigcup_{\alpha \in G(\gamma)} \Delta\left(S_{\alpha}\right)$ satisfying the following properties: For each $k=0,1, \ldots$,

${ }^{7}$ Although $G(\gamma)$ is a subset of the lattice of all restricted games given $\gamma$, and although the partial order on $G(\gamma)$ is the partial order of the lattice of all restricted games, $G(\gamma)$ is not necessarily a sublattice of the lattice of all restricted games. This is because for example $\alpha, \beta \in G(\gamma), \alpha \vee \beta$, might not be in $G(\gamma)$, but only a game larger than $\alpha \vee \beta$. That is, the join of $\alpha$ and $\beta$ in $G(\gamma)$ may not coincide with the join of $\alpha$ and $\beta$ in the lattice of all restricted games given $\gamma$. 
(i') If $\omega=\left(\alpha_{j}\right)_{j \in I^{0}} \in S_{\alpha}$ then $t_{i}^{k}(\omega) \in \Delta\left(S_{\alpha_{i}}\right)$. (Note that $\alpha_{i} \preceq \alpha$.)

(ii') If $\omega=\left(\beta_{j}\right)_{j \in I^{0}} \in S_{\alpha}, t_{i}^{k}(\omega) \in \Delta\left(S_{\beta_{i}}\right), \alpha \succeq \beta_{i} \succeq \delta$, then $t_{i}^{k}\left(\omega_{\delta}\right)=t_{i}^{k}(\omega)_{\mid S_{\delta}}$.

These properties imply properties (i) to (iii) of type mappings in Definition 1 (see Appendix C for a proof):

Lemma 1 For each $k=0,1, \ldots$, the type mapping satisfies the properties (i) to (iii) of Definition 1. More specific, ( $\left.i^{\prime}\right)$ implies (i), (ii') implies (ii), and ( $\left.i^{\prime}\right)$ implies (iii).

Let $\bar{\alpha}:=\left(\alpha_{i}\right)_{i \in I^{0}}$ with $\alpha_{i}=\alpha \in G(\gamma)$ for all $i \in I^{0}$. That is, a bar over $\alpha$ signifies a "monomorphic" state in which all components are identical to $\alpha$. Note that by Property (i'), for all $\alpha \in G(\gamma), t_{i}^{k}(\bar{\alpha}) \in \Delta\left(S_{\alpha}\right)$ for all $i \in I$ and $k=0,1, \ldots$

In the current context we impose additional properties. For each $i \in I$ and each $k=0,1, \ldots$,

(iv') If $\omega=\left(\alpha_{i}\right)_{i \in I^{0}}$ and $\omega^{\prime}=\left(\beta_{i}\right)_{i \in I^{0}}$ with $\alpha_{i}=\beta_{i}$, then $t_{i}^{k}(\omega)=t_{i}^{k}\left(\omega^{\prime}\right)$.

Property (iv') states that if at two states player $i$ has the same awareness, then she has the same beliefs at those two states. That is, for each awareness level, each agent has only one type. Although this is not necessary, it simplifies our analysis. Together with (ii'), it implies that Introspection (Property (iv)) is satisfied.

Let $G^{*}(\gamma)=\left\{\alpha \in G(\gamma):\left|M_{i}^{\alpha}\right| \geq 2\right.$ for all $\left.i \in I\right\}$. This is the subset of restricted games in which every player has besides the option of "doing nothing" also an action of the original game. The next two assumptions are conceptually more important. For each $i \in I$ and each $k=0,1, \ldots$,

(v) Uncertainty about opponents' awareness: $t_{i}^{k}(\bar{\gamma})(\{\omega\})>0$ if and only if $\omega=$ $\left(\alpha_{j}\right)_{j \in I^{0}} \in S_{\gamma}$ is such that $\alpha_{i}=\gamma$ and $\beta_{j} \in G^{*}(\gamma)$ for all $j \in I \backslash\{i\}$.

Uncertainty about opponents' awareness, Property (v), implies that at the objective true state $\bar{\gamma}$ player $i$ is uncertain about which nonempty subset of actions in the original game opponents are aware of. It also implies that at the objective true state $\bar{\gamma}$ player $i$ is certain that each opponent is aware of at least some action in the original game. Ultimately we are interested in the robustness of equilibria in which players play actions of the original game instead being forced to play their default action. Property (iv') implies that property (v) holds also for all states in the upmost space in which player $i$ is fully aware.

Finally, Property (vi) below, certainty in the limit, just means that in the limit at the objective true state $\bar{\gamma}$ there is common certainty that all players are aware of all actions. That is, in the limit the players at state $\bar{\gamma}$ are commonly certain that they are playing the strategic game $\gamma$. 
(vi) Certainty in the limit: For every $i \in I, \lim _{k \rightarrow \infty} t_{i}^{k}(\bar{\gamma})(\{\omega\})$ exists for all $\omega \in S_{\gamma}$, and $t_{i}^{k}(\bar{\gamma})(\{\bar{\gamma}\}) \rightarrow 1$ as $k \rightarrow \infty$.

For each $i \in I$, define a correspondence $\mathcal{M}_{i}: \Omega \longrightarrow 2^{M_{i}}$ by $\mathcal{M}_{i}(\omega)=M_{i}^{\alpha}$ if $\omega \in S_{\alpha}$, where $M_{i}^{\alpha}$ is player $i$ 's set of actions in the game $\alpha \in G(\gamma)$.

Finally, for each player $i \in I$, define a utility function $u_{i}: \bigcup_{\omega \in \Omega}\left(\prod_{j \in I} \mathcal{M}_{j}(\omega)\right) \times$ $\{\omega\} \longrightarrow \mathbb{R}$ in the following manner: For all $\alpha \in G(\gamma)$, if $\omega \in S_{\alpha}$ then $u_{i}(\cdot, \omega)=\left.v_{i}\right|_{M^{\alpha}}$ where $M^{\alpha}$ is the action space in game $\alpha$.

With these definitions we have (see Appendix C for a proof):

Remark 3 For each finite strategic game $\gamma$ and each $k=0,1, \ldots$ we have that

$$
\Gamma\left(\underline{\mathcal{S}}_{\gamma}^{k}\right):=\left\langle\mathcal{S}_{\gamma},\left(r_{\beta}^{\alpha}\right)_{\alpha \succeq \beta ; \alpha, \beta \in G(\gamma)},\left(t_{i}^{k}\right)_{i \in I},\left(M_{i}\right)_{i \in I},\left(\mathcal{M}_{i}\right)_{i \in I},\left(u_{i}\right)_{i \in I}\right\rangle
$$

is a finite Bayesian game with unawareness.

Proposition 1 and Remark 3 imply:

Corollary 1 For each finite strategic game $\gamma$ and each $k=0,1, \ldots$ there exists a Bayesian Nash equilibrium of $\Gamma\left(\underline{\mathcal{S}}_{\gamma}^{k}\right)$.

Denote by $\sigma^{k}=\left(\left(\sigma_{i}^{k}(\omega)\right)_{i \in I}\right)_{\omega \in \Omega}$ a strategy combination in the game $\Gamma\left(\underline{\mathcal{S}}_{\gamma}^{k}\right)$. Moreover, we denote by $E\left(\Gamma\left(\underline{\mathcal{S}}_{\gamma}^{k}\right)\right)$ the set of Bayesian Nash equilibria of the game $\Gamma\left(\underline{\mathcal{S}}_{\gamma}^{k}\right)$.

The next remark follows from the fact that for each player $i \in I$, the action $d_{i}$ is strictly dominated by strategies $\tilde{M}_{i}$ in the original game $\tilde{\gamma}$.

Remark 4 If $\sigma^{k} \in E\left(\Gamma\left(\underline{\mathcal{S}}_{\gamma}^{k}\right)\right)$ then for every player $i \in I$, the equilibrium strategy $\sigma_{i}^{k}(\bar{\gamma})$ assigns probability zero to the default action $d_{i}$. Hence, we consider $\sigma_{i}^{k}(\bar{\gamma})$ as a probability measure on player $i$ 's set of actions $\tilde{M}_{i}$ in the original game $\tilde{\gamma}$.

The next lemma says that the limit at state $\bar{\gamma}$ of a convergent sequence of Bayesian Nash equilibria of games $\Gamma\left(\underline{\mathcal{S}}_{\gamma}^{k}\right)$ as $k \rightarrow \infty$ is an equilibrium of the original game $\tilde{\gamma}$. (See Appendix C for a proof.)

Lemma 2 If $\sigma^{k} \in E\left(\Gamma\left(\underline{\mathcal{S}}_{\gamma}^{k}\right)\right)$ and $\sigma^{k}(\bar{\gamma}) \rightarrow \nu$ as $k \rightarrow \infty$, then $\nu$ is an equilibrium of $\tilde{\gamma}$.

Definition 7 (Unawareness Perfect Equilibrium) An Unawareness Perfect Equilibrium $\nu$ of the strategic game $\tilde{\gamma}$ is a Nash equilibrium of $\tilde{\gamma}$ for which there exists a sequence of Bayesian games with unawareness $\left(\Gamma\left(\underline{\mathcal{S}}_{\gamma}^{k}\right)\right)_{k=0}^{\infty}$ (as defined above, with certainty about the players' awareness of actions in the limit) with Bayesian Nash equilibria $\sigma^{k} \in E\left(\Gamma\left(\underline{\mathcal{S}}_{\gamma}^{k}\right)\right), k=0,1, \ldots$, for which $\sigma^{k}(\bar{\gamma}) \rightarrow \nu$ as $k \rightarrow \infty$. 
That is, an Unawareness Perfect Equilibrium of a strategic game is a Nash equilibrium that is robust to uncertainty over unawareness of actions as this uncertainty vanishes.

Using Lemma 2 and the existence result for Bayesian Nash equilibrium (Proposition 1), we prove in the appendix the following:

Theorem 1 For every finite strategic game, an Unawareness Perfect Equilibrium exists.

It turns out that unawareness perfection is closely related to undominated actions. An action $m_{i} \in \tilde{M}_{i}$ is weakly dominated in the original game $\tilde{\gamma}$ if there exists a mixed action $\mu_{i} \in \Delta\left(\tilde{M}_{i}\right)$ such that $\tilde{v}_{i}\left(\mu_{i}, m_{-i}\right) \geq \tilde{v}_{i}\left(m_{i}, m_{-i}\right)$ for all $m_{-i} \in \tilde{M}_{-i}$ and $\tilde{v}_{i}\left(\mu_{i}, m_{-i}\right)>$ $\tilde{v}_{i}\left(m_{i}, m_{-i}\right)$ for some $m_{-i} \in \tilde{M}_{-i}$. This is the standard definition. An action that is not weakly dominated is undominated. A Nash equilibrium is undominated if every player's (mixed) equilibrium action assigns strict positive probability to undominated actions only. Note that we focus on undominated Nash equilibrium of the original game $\tilde{\gamma}$. Since we allow a player's payoffs to be arbitrary if an opponent takes the default action, it is not generally the case that if an action is undominated in the original game $\tilde{\gamma}$, then it is also undominated in the augmented game $\gamma$. We prove in the appendix the following characterization:

Theorem 2 A Nash equilibrium of a finite strategic game is an Unawareness Perfect Equilibrium if and only if it is undominated (i.e., not weakly dominated).

That every Unawareness Perfect Equilibrium is undominated follows essentially from continuity of von Neumann-Morgenstern utilities and the fact that it is a limit of Bayesian Nash equilibria in which opponents' types may be forced to play any non-default action due to unawareness of alternative actions. Any action played with strict positive probability in an unawareness perfect equilibrium of a strategic game must also get strict positive probability in a Bayesian Nash equilibrium of $\Gamma\left(S_{\gamma}^{k}\right)$ at some point during the sequence for sufficiently large $k$. This implies that the action is a best reply against a full support belief over all action profiles that do not contain the default actions. Hence, by Pearce (1984, Lemma 4) the action is undominated in the original game. The proof of the converse is more involved as we construct a sequence of Bayesian games in which we mimic the full support belief that rationalizes a player's undominated equilibrium strategy by beliefs about opponents' restricted awareness and their play restricted to actions that they are aware of. Any undominated Nash equilibrium is a best reply against the opponents' equilibrium actions and again by Pearce (1984, Lemma 4), a full support belief over opponents' actions. We show that for any undominated Nash equilibrium of a finite strategic game we can construct a sequence of Bayesian games with unawareness with a corresponding sequence of Bayesian Nash equilibria. Any fully aware player's Bayesian equilibrium action is a best reply to possibly unaware opponent's Bayesian equilibrium strategies and, in the limit, to the mixed strategies of the others in an equilibrium of the true strategic game. 
For games with more than two players, unawareness perfection is weaker than trembling hand perfection by Selten (1975). Since trembling hand perfect equilibria and undominated equilibria coincide in two-player games (see van Damme, 1991), Theorem 2 implies that unawareness perfect equilibria and trembling hand perfect equilibria coincide in two-player games. There are assumptions embodied in trembling hand perfect equilibrium that have bite in games with more than two players. First, in a trembling hand perfect equilibrium any two players have common knowledge about the nature of trembles of a third player. Second, trembles are made independently. Both assumptions could be cast analogously in our framework. Any two players could have common belief about a third player's awareness of actions. Moreover, types may be independent. We do not know what would justify such assumptions. Thus, we content ourselves with studying just robustness of equilibria to small uncertainty about awareness of actions without additional assumptions. As it turns out, this justifies ruling out dominated equilibria but no more than that.

\section{$5 \quad$ Related Literature}

There is a growing literature on unawareness both in economics and computer science. ${ }^{8}$ The independent parallel work of Sadzik (2006) is closest to ours. Building to a certain extent on our earlier work, Heifetz, Meier, and Schipper (2006), he presents a framework of unawareness with probabilistic beliefs in which the common prior on the upmost space is a primitive. In contrast, we take types as primitives and a prior may be defined on the entire unawareness belief structure as a convex combination of the type's beliefs (see Heifetz, Meier, and Schipper, 2013a). Sadzik (2006) also considers Bayesian games with unawareness, but his definition of Bayesian strategy and consequently the notion of equilibrium differs from ours. As argued above, we do not confine actions of a type with a narrow horizon to be some average of actions of the corresponding types with a wider horizon, a restriction made in Sadzik (2006). As a result, in our notion of Bayesian equilibrium every type maximizes and is certain that every other type that she is aware of maximizes as well, while in the equilibrium of actions proposed in Sadzik (2006) a type may believe that another player is irrational. Sadzik (2006) does not allow for players to be unaware of other players, while we do.

A purely syntactic framework with unawareness is presented by Feinberg (2005) which he applies to games with unawareness of actions but complete information. In Section 3, we discuss an interesting example due to Feinberg (2005) and demonstrate that higher order awareness of unawareness in Feinberg (2005) corresponds to higher order belief of unawareness in our model. In a more recent paper, Feinberg (2012) discusses games with unawareness and incomplete information. He explicitly models many views of a game, each (mutual) view being a finite sequence of player names $i_{1}, \ldots, i_{n}$ with the interpretation that this is how $i_{1}$ views how .... how $i_{n}$ views the game. This differs from

\footnotetext{
${ }^{8}$ See http://www.econ.ucdavis.edu/faculty/schipper/unaw.htm for a bibliography.
} 
our unawareness belief structures in which each state "encapsulates" the views of the players, their views about other players' views etc. in a parsimonious way familiar from standard structures. Feinberg (2012) also extends Bayesian Nash equilibrium to games with unawareness and incomplete information.

In a framework similar to Feinberg (2005, 2012), Copič and Galeotti (2006) study two-player games with either unawareness of actions or unawareness of types (with a prior as a primitive). Yet, their notion of equilibrium differs from Bayesian equilibrium because the authors require that in equilibrium beliefs over actions and payoffs must correspond to the true joint distribution over own payoffs and the opponent's actions.

We strictly prefer to interpret our Bayesian games with unawareness as static games because dynamic strategic interaction under unawareness poses further complications such as changes of awareness. Halpern and Rêgo (2013), Heifetz, Meier, and Schipper (2011, 2013b), Rêgo and Halpern (2012), Li (2006), Ozbay (2007), Meier and Schipper (2012), Grant and Quiggin (2013), and Feinberg (2012) present models of extensive-form games with unawareness and discuss solution concepts.

\section{A Bayesian Games with Unawareness: Allowing for Unawareness of Players}

So far, we did not allow for unawareness of players. In standard Bayesian game theory, ignorance of players is modeled by dummy players. This is distinct from being unable to conceive of a player at all. In this subsection we allow for unawareness of players. This requires that we generalize our interactive unawareness belief structure such that a player may exist only at some states but not at others. Such a generalization may be useful to extensions to psychological games with unawareness (see Nielsen and Sebald, 2011). For instance, being aware of an observer may affect behavior even if the observer has no active role in the game. This is known in psychology as the observer effect.

Definition 8 A Bayesian game with unawareness (that also allows for unawareness of players) is a tuple

$$
\Gamma(\underline{\mathcal{S}}):=\left\langle\mathcal{S},\left(r_{S_{\beta}}^{S_{\alpha}}\right)_{S_{\beta} \preceq S_{\alpha}}, \mathcal{E},\left(t_{i}\right)_{i \in I},\left(M_{i}\right)_{i \in I},\left(\mathcal{M}_{i}\right)_{i \in I},\left(u_{i}\right)_{i \in I}\right\rangle
$$

defined as follows:

(0) $\mathcal{S}=\left\{S_{\alpha}\right\}_{\alpha \in \mathcal{A}}$ is as before a complete lattice of spaces with surjective and commuting projections $\left(r_{S_{\beta}}^{S_{\alpha}}\right)$, for $S_{\beta} \preceq S_{\alpha}$ (see Section 2).

(i) $\mathcal{E}: I \longrightarrow \Sigma$ is the "existence" correspondence that assigns to each player $i \in I$ an event in which she exists. Moreover, $\mathcal{S}_{i}:=\{S \in \mathcal{S}: \mathcal{E}(i) \cap S \neq \emptyset\}$ is the complete sublattice of spaces with states in which player $i$ exists. 
(ii) For every player $i \in I, t_{i}: \mathcal{E}(i) \longrightarrow \bigcup_{S \in \mathcal{S}_{i}} \Delta(S)$ is a type mapping that satisfies Properties (i) to (iii) (see Section 2) such that for every $\omega \in \mathcal{E}(i), t_{i}(\omega)(\mathcal{E}(i))=1$.

(iii) For every $i \in I, M_{i}$ is a nonempty finite set of actions. $\mathcal{M}_{i}: \mathcal{E}(i) \longrightarrow 2^{M_{i}} \backslash\{\emptyset\}$ is a correspondence, for $i \in I$, with the following properties:

a.) For every $M_{i}^{\prime}$ with $\emptyset \neq M_{i}^{\prime} \subseteq M_{i}$ : If there is a state $\omega$ with $\omega \in \mathcal{E}(i)$, then the set $\left\{\omega^{\prime} \in \Omega: M_{i}^{\prime} \subseteq \mathcal{M}_{i}\left(\omega^{\prime}\right)\right\}$ is an event.

b.) If $\omega \in \mathcal{E}(i)$ and $\omega^{\prime}, \omega^{\prime \prime} \in S_{t_{i}(\omega)} \cap\left[t_{i}(\omega)\right]$, then $\mathcal{M}_{i}\left(\omega^{\prime}\right)=\mathcal{M}_{i}\left(\omega^{\prime \prime}\right)$.

(iv) Further, we impose introspection as follows: For $\omega \in \mathcal{E}(i)$,

$$
t_{i}(\omega)\left(\left\{\omega^{\prime} \in \mathcal{E}(i): t_{i}\left(\omega^{\prime}\right)_{\mid S_{t_{i}(\omega)}}=t_{i}(\omega)\right\}\right)=1 .
$$

(v) For $i \in I, u_{i}: \bigcup_{\omega \in \mathcal{E}(i)}\left(\left(\prod_{j \in I(\omega)} \mathcal{M}_{j}(\omega)\right) \times\{\omega\}\right) \longrightarrow \mathbb{R}$ is the utility function of $i$, where $I(\omega):=\{i \in I: \omega \in \mathcal{E}(i)\}$.

This game allows for unawareness of events, actions, outcomes, and players. For every player $i \in I$, the "existence" correspondence $\mathcal{E}$ assigns to $i$ the event in which she exists. Consequently we restrict player $i$ 's type mapping to states at which she exists. Moreover, player $i$ 's type is concentrated only on states in which she exists. A player can not assign strict positive probability to states at which she does not exist. The correspondence $\mathcal{M}_{i}$ assigns a non-empty set of actions for player $i$ only to the set of states in which player $i$ exists. The dimension of the domain of a utility function may vary from state to state, since players may exist in some states but not in others, and each players utility at a state depends on the actions of all the players that exist in that state.

Note that if $\mathcal{E}(i)=\Omega$ for all $i \in I$, then we obtain an unawareness belief structure and a Bayesian game with unawareness as defined before.

Note further that if $\omega \in \mathcal{E}(i)$, then $\left[t_{i}(\omega)\right]:=\left\{\omega^{\prime} \in \Omega: t_{i}\left(\omega^{\prime}\right)=t_{i}(\omega)\right\} \subseteq \mathcal{E}(i)$.

A strategy of player $i$ is now adapted to the event in which she exists.

Definition 9 A strategy of player $i$ in a Bayesian game with unawareness is a function $\sigma_{i}: \mathcal{E}(i) \longrightarrow \Delta\left(M_{i}\right)$ such that for all $\omega \in \mathcal{E}(i)$,

(i) $\sigma_{i}(\omega) \in \Delta\left(\mathcal{M}_{i}\left(\omega_{S_{t_{i}}(\omega)}\right)\right)$.

(ii) $t_{i}\left(\omega^{\prime}\right)=t_{i}(\omega)$ implies $\sigma_{i}\left(\omega^{\prime}\right)=\sigma_{i}(\omega)$.

Denote $\sigma_{S_{t_{i}(\omega)}}:=\left(\left(\sigma_{j}\left(\omega^{\prime}\right)\right)_{j \in I\left(\omega^{\prime}\right)}\right)_{\omega^{\prime} \in S_{t_{i}(\omega)}}$. The expected utility of player-type $\left(i, t_{i}(\omega)\right)$ from the strategy profile $\sigma_{S_{t_{i}(\omega)}}$ is given by

$U_{\left(i, t_{i}(\omega)\right)}\left(\sigma_{S_{t_{i}(\omega)}}\right):=$ 


$$
\sum_{\omega^{\prime} \in S_{t_{i}(\omega)}} \sum_{m \in \prod_{j \in I\left(\omega^{\prime}\right)} \mathcal{M}_{j}\left(\omega_{S_{t_{j}\left(\omega^{\prime}\right)}^{\prime}}\right)}\left(\prod_{j \in I\left(\omega^{\prime}\right)} \sigma_{j}\left(\omega^{\prime}\right)\left(\left\{m_{j}\right\}\right)\right) \cdot u_{i}\left(\left(m_{j}\right)_{j \in I\left(\omega^{\prime}\right)}, \omega^{\prime}\right) t_{i}(\omega)\left(\left\{\omega^{\prime}\right\}\right) .
$$

This expression is analogous to equation (1) except that the set of players $I$ is now replaced by $I\left(\omega^{\prime}\right)$.

Definition 5 and Proposition 1 are now generalized to:

Definition 10 (Equilibrium) Given a Bayesian game with unawareness $\Gamma(\underline{\mathcal{S}})$, define the associated strategic game by

(i) $\left\{\left(i, t_{i}(\omega)\right): \omega \in \Omega\right.$ and $\left.i \in I(\omega)\right\}$ is the set of players,

and for each player $\left(i, t_{i}(\omega)\right)$,

(ii) the set of mixed strategies is $\Delta\left(\mathcal{M}_{i}\left(\omega_{S_{t_{i}}(\omega)}\right)\right.$, and

(iii) the utility function is given by Equation (2).

A profile $\left(\sigma_{i}\right)_{i \in I}$ is an equilibrium of the Bayesian game with unawareness if and only if the following is an equilibrium of the associated strategic game: $\left(i, t_{i}(\omega)\right)$ plays $\sigma_{i}(\omega)$, for all $i \in I(\omega)$ and $\omega \in \Omega$.

Proposition 3 (Existence) Let $\Gamma(\underline{\mathcal{S}})=\left\langle\mathcal{S},\left(r_{S_{\beta}}^{S_{\alpha}}\right)_{S_{\beta} \preceq S_{\alpha}}, \mathcal{E},\left(t_{i}\right)_{i \in I},\left(M_{i}\right)_{i \in I},\left(\mathcal{M}_{i}\right)_{i \in I},\left(u_{i}\right)_{i \in I}\right\rangle$ be a Bayesian game with unawareness. If $I, \Omega$, and $\left(M_{i}\right)_{i \in I}$ are finite, then there exists an equilibrium.

Proof. By Nash's (1950) theorem.

Recall $l(S):=\left\{S^{\prime} \in \mathcal{S}: S^{\prime} \preceq S\right\}$. Definition 6, Proposition 2, and Remark 1 are now generalized, respectively, as follows:

Definition 11 Given a Bayesian game with unawareness

$$
\Gamma(\underline{\mathcal{S}})=\left\langle\mathcal{S},\left(r_{S_{\beta}}^{S_{\alpha}}\right)_{S_{\beta} \preceq S_{\alpha}}, \mathcal{E},\left(t_{i}\right)_{i \in I},\left(M_{i}\right)_{i \in I},\left(\mathcal{M}_{i}\right)_{i \in I},\left(u_{i}\right)_{i \in I}\right\rangle,
$$

we can define for any $S^{\prime} \in \mathcal{S}$ an $S^{\prime}$-partial Bayesian game with unawareness

$$
\Gamma\left(\underline{l\left(S^{\prime}\right)}\right)=\left\langle l\left(S^{\prime}\right),\left(r_{S_{\beta}}^{S_{\alpha}}\right)_{S_{\beta} \preceq S_{\alpha} \preceq S^{\prime}}, \mathcal{E}^{\prime},\left(t_{i}\right)_{i \in I\left(\Omega^{\prime}\right)},\left(M_{i}\right)_{i \in I\left(\Omega^{\prime}\right)},\left(\mathcal{M}_{i}^{\prime}\right)_{i \in I\left(\Omega^{\prime}\right)},\left(u_{i}\right)_{i \in I\left(\Omega^{\prime}\right)}\right\rangle,
$$

in which $\mathcal{E}^{\prime}(i)=\mathcal{E}(i) \cap \Omega^{\prime}$, where $\Omega^{\prime}=\bigcup_{S^{\prime \prime} \in l\left(S^{\prime}\right)} S^{\prime \prime}$, and for any $i \in I\left(\Omega^{\prime}\right):=\bigcup_{\omega \in \Omega^{\prime}} I(\omega)$, $\mathcal{M}_{i}^{\prime}$ is $\mathcal{M}_{i}$ restricted to $\mathcal{E}^{\prime}(i)$. 
Proposition 4 ("Upwards Induction") Given a Bayesian game with unawareness $\left\langle\mathcal{S},\left(r_{S_{\beta}}^{S_{\alpha}}\right)_{S_{\beta} \preceq S_{\alpha}}, \mathcal{E},\left(t_{i}\right)_{i \in I},\left(M_{i}\right)_{i \in I},\left(\mathcal{M}_{i}\right)_{i \in I},\left(u_{i}\right)_{i \in I}\right\rangle$, consider for $S^{\prime}, S^{\prime \prime} \in \mathcal{S}$ with $S^{\prime} \preceq$ $S^{\prime \prime}$ the $S^{\prime}$-partial (resp. $S^{\prime \prime}$-partial) Bayesian game with unawareness. If $I, \Omega$, and $\left(M_{i}\right)_{i \in I}$ are finite, then for every equilibrium of the $S^{\prime}$-partial Bayesian game, there is an equilibrium of the $S^{\prime \prime}$-partial Bayesian game in which equilibrium strategies of playertypes in $\left\{\left(i, t_{i}(\omega)\right): \omega \in \Omega^{\prime}=\bigcup_{S \in l\left(S^{\prime}\right)} S\right.$ and $\left.i \in I\left(\Omega^{\prime}\right)\right\}$ are identical with the equilibrium strategies in the $S^{\prime}$-partial Bayesian game.

Proof. Let $\sigma_{\mid \Omega^{\prime}}^{*}$ be an equilibrium in the $S^{\prime}$-partial Bayesian game with unawareness $\Gamma\left(\underline{\mathcal{S}}^{\prime}\right)$. For $S^{\prime \prime} \succeq S^{\prime}$ we define a strategic form game with

- $I\left(\Omega^{\prime \prime} \backslash \Omega^{\prime}\right):=\left\{\left(i, t_{i}(\omega)\right): \omega \in \Omega^{\prime \prime}, i \in I(\omega)\right\} \backslash\left\{\left(i, t_{i}(\omega)\right): \omega \in \Omega^{\prime}, i \in I(\omega)\right\}$ being the set of players,

- the set of strategies of player $\left(i, t_{i}(\omega)\right) \in I\left(\Omega^{\prime \prime} \backslash \Omega^{\prime}\right)$ is $\Delta\left(\mathcal{M}_{i}\left(\omega_{S_{t_{i}(\omega)}}\right)\right),{ }^{9}$

- the payoff function of player $\left(i, t_{i}(\omega)\right)$ is given by equation (1) but fixing the strategy of each (dummy) player in $\left\{\left(i, t_{i}\left(\omega^{\prime}\right)\right): \omega^{\prime} \in \Omega^{\prime}, i \in I\left(\omega^{\prime}\right)\right\}$ to her respective equilibrium strategy $\sigma_{i}^{*}(\omega)$ of the $S^{\prime}$-partial Bayesian game with unawareness $\Gamma\left(\underline{\mathcal{S}}^{\prime}\right)$.

Since $I, \Omega$, and $\left(M_{i}\right)_{i \in I}$ are finite, this strategic game has an equilibrium by Nash's (1950) theorem. Fix one equilibrium of this game.

Consider now the strategy profile $\sigma_{\mid \Omega^{\prime \prime}}^{*}$ in which players in $\left\{\left(i, t_{i}(\omega)\right): \omega \in \Omega^{\prime}, i \in I(\omega)\right\}$ play their component of the profile $\sigma_{\mid \Omega^{\prime}}^{*}$ and players in $I\left(\Omega^{\prime \prime} \backslash \Omega^{\prime}\right)$ play the equilibrium strategies of the equilibrium in the above defined strategic game.

We need to show that $\sigma_{\mid \Omega^{\prime \prime}}^{*}$ is an equilibrium of the $S^{\prime \prime}$-partial Bayesian game with unawareness $\Gamma\left(\underline{\mathcal{S}}^{\prime \prime}\right)$. Suppose not, then for some player $\left(i, t_{i}(\omega)\right) \in I\left(\Omega^{\prime \prime}\right)=\left\{\left(i, t_{i}\left(\omega^{\prime}\right)\right)\right.$ : $\left.\omega^{\prime} \in \Omega^{\prime \prime}, i \in I\left(\omega^{\prime}\right)\right\}$ there exists $\sigma_{i}(\omega) \in \Delta\left(\mathcal{M}_{i}\left(\omega_{S_{t_{i}}(\omega)}\right)\right)$ with $\sigma_{i}(\omega) \neq \sigma_{i}^{*}(\omega)$ such that for $\sigma_{S_{t_{i}(\omega)}}:=\left(\sigma_{i}(\omega),\left(\sigma_{j}^{*}\left(\omega^{\prime}\right)\right)_{\omega^{\prime} \in S_{t_{i}(\omega), j \in I(\omega) \backslash\{i\}}}\right)$ we have

$$
U_{\left(i, t_{i}(\omega)\right)}\left(\sigma_{S_{t_{i}(\omega)}}\right)>U_{\left(i, t_{i}(\omega)\right)}\left(\sigma_{S_{t_{i}(\omega)}}^{*}\right),
$$

i.e., there exists a profitable deviation from $\sigma_{\mid \Omega^{\prime \prime}}^{*}$ for some player-type $\left(i, t_{i}(\omega)\right)$ with $\omega \in \Omega^{\prime \prime}$ and $i \in I(\omega)$ given that all other player-types in $I\left(\Omega^{\prime \prime}\right)$ play their equilibrium strategy.

If $\left(i, t_{i}(\omega)\right) \in I\left(\Omega^{\prime \prime} \backslash \Omega^{\prime}\right)$ then her strategy is not an equilibrium strategy in the above defined strategic game, a contradiction. If $\left(i, t_{i}(\omega)\right) \in\left\{\left(i, t_{i}(\omega)\right): \omega^{\prime} \in \Omega^{\prime}, i \in I\left(\omega^{\prime}\right)\right\}$, then since her payoffs are identical in both games, her strategy is not an equilibrium

${ }^{9}$ Note that for $\left(i, t_{i}(\omega)\right) \in I\left(\Omega^{\prime \prime} \backslash \Omega^{\prime}\right)$ we have either $S_{t_{i}(\omega)} \succ S^{\prime}$ or $S_{t_{i}(\omega)}$ and $S^{\prime}$ are incomparable. 
strategy in the $S^{\prime}$-partial Bayesian game with unawareness $\Gamma\left(\underline{\mathcal{S}}^{\prime}\right)$, a contradiction. Hence $\sigma_{\mid \Omega^{\prime \prime}}^{*}$ must be an equilibrium of the $S^{\prime \prime}$-partial Bayesian game with unawareness $\Gamma\left(\underline{\mathcal{S}}^{\prime \prime}\right)$.

It is easy to construct examples of Bayesian games with unawareness in which unaware players may have commitment power and the "value of awareness" may be negative. For instance, in a simultaneous-move linear Cournot duopoly, a player who is unaware of his opponent can obtain the Stackelberg leader profit if the opponent knows that the first player is unaware of him.

The converse to Proposition 4 follows from the consistency of Nash equilibrium (see Peleg and Tijs, 1996, and Peleg, Potters, and Tijs, 1996):

Remark $5 \operatorname{Let}\left\langle\mathcal{S},\left(r_{S_{\beta}}^{S_{\alpha}}\right)_{S_{\beta} \preceq S_{\alpha}}, \mathcal{E},\left(t_{i}\right)_{i \in I},\left(M_{i}\right)_{i \in I},\left(\mathcal{M}_{i}\right)_{i \in I},\left(u_{i}\right)_{i \in I}\right\rangle$ be a Bayesian game with unawareness. Consider for $S^{\prime}, S^{\prime \prime} \in \mathcal{S}$ with $S^{\prime} \preceq S^{\prime \prime}$ the $S^{\prime}$-partial (resp. $S^{\prime \prime}$-partial) Bayesian game with unawareness. Then for every equilibrium of the $S^{\prime \prime}$-partial Bayesian game there is a unique equilibrium of the $S^{\prime}$-partial Bayesian game in which the equilibrium strategies of player-types in $\left\{\left(i, t_{i}(\omega)\right): \omega \in \Omega^{\prime}=\bigcup_{S \in l\left(S^{\prime}\right)} S\right.$ and $\left.i \in I\left(\Omega^{\prime}\right)\right\}$ are identical to the equilibrium strategies of the $S^{\prime \prime}$-partial Bayesian game.

\section{B Connection to Standard Bayesian Games}

In this section we compare Bayesian games with unawareness to standard Bayesian games. In particular, we show how to derive a standard type space with zero probability from our unawareness structure by "flattening" our lattice of spaces. "Flattening" the game is a purely technical procedure. While we can show a correspondence between equilibria in a Bayesian game with unawareness and equilibria in a standard Bayesian game, the equilibrium in the standard Bayesian game cannot be interpreted anymore under unawareness because the "language" required to identify events of which a player could be unaware is essentially "erased". Since a flattened structure is a standard type-space, the "Dekel-Lipman-Modica-Rustichini critique" applies (Modica and Rustichini, 1994, Dekel, Lipman, and Rustichini, 1998). Hence unawareness is trivial in the flattened game.

Let $\underline{\mathcal{S}}$ be an unawareness belief structure. We define the flattened type-space associated with the unawareness belief structure $\underline{\mathcal{S}}$ by

$$
F(\underline{\mathcal{S}}):=\left\langle\Omega,\left(t_{i}^{F}\right)_{i \in I}\right\rangle
$$

where $\Omega$ is the union of all state-spaces in the unawareness belief structure $\underline{\mathcal{S}}$ and $t_{i}^{F}$ : $\Omega \longrightarrow \Delta(\Omega)$ is defined by,

$$
t_{i}^{F}(\omega)(E):= \begin{cases}t_{i}(\omega)\left(E \cap S_{t_{i}(\omega)}\right) & \text { if } E \cap S_{t_{i}(\omega)} \neq \emptyset \\ 0 & \text { otherwise. }\end{cases}
$$

Note that when flattening an unawareness belief structure, we lose the lattice structure of spaces and thus the event structure. For instance, recall that in an unawareness 
belief structure the negation of the event $\left(D^{\uparrow}, S\right)$ with $D \subseteq S$ is defined by $\neg\left(D^{\uparrow}, S\right)=$ $\left((S \backslash D)^{\uparrow}, S\right)$. This is typically a proper subset of the complement $\Omega \backslash D^{\uparrow}$. That is, $(S \backslash D)^{\uparrow} \varsubsetneqq \Omega \backslash D^{\uparrow}$. But it is precisely our lattice structure that allows us to circumvent the impossibility result by Dekel, Lipman, and Rustichini (1998).

A standard type-space on $Y$ for the player set $I$ is a tuple

$$
\underline{Y}:=\left\langle Y,\left(t_{i}\right)_{i \in I}\right\rangle,
$$

where $Y$ is a nonempty set and for $i \in I, t_{i}$ is a function from $Y$ to $\Delta(Y)$, the space of countably additive probability measures on $Y$, such that for all $\omega \in Y, t_{i}(\omega)\left(\left[t_{i}(\omega)\right]\right)=1$ with $\left[t_{i}(\omega)\right]:=\left\{\omega^{\prime} \in Y: t_{i}\left(\omega^{\prime}\right)=t_{i}(\omega)\right\}$ (i.e., introspection).

The properties of the type mapping in the unawareness belief structure $\mathcal{S}$ implies immediately the following:

Proposition 5 If $\underline{\mathcal{S}}$ is an unawareness belief structure, then $F(\underline{\mathcal{S}})$ is a standard typespace. Moreover, it has the following property: For every $p>0, E \subseteq \Omega$, and $i \in I$ : $\left\{\omega \in \Omega: t_{i}(\omega)\left(E \cap S_{t_{i}(\omega)}\right) \geq p\right\}=\left\{\omega \in \Omega: t_{i}^{F}(\omega)(E) \geq p\right\}$.

Proof. We only have to show introspection. I.e., for all $\omega \in \Omega, i \in I$, and $E \in \mathcal{F}$, $t_{i}^{F}(\omega)\left(\left[t_{i}^{F}(\omega)\right]\right)=1$ with $\left[t_{i}^{F}(\omega)\right]=\left\{\omega^{\prime} \in \Omega: t_{i}^{F}\left(\omega^{\prime}\right)=t_{i}^{F}(\omega)\right\}$. But this follow directly from property (iv) of the type mapping in the unawareness belief structure $\mathcal{S}$.

A flattened unawareness structure is just a standard type-space. To derive such a type-space, one extends a player's type mapping by assigning probability zero to sets for which the player's belief was previously undefined. Of course, once an unawareness structure is flattened, there is no way to analyze reasoning about unawareness anymore since by Dekel, Lipman, and Rustichini (1998) unawareness is trivial.

Note that the converse to Proposition 5 is not true. I.e., given a standard type-space, it is not always possible to find some unawareness structure with non-trivial unawareness. For instance, let $X=\left\{\omega_{1}, \omega_{2}, \omega_{3}\right\}$ with $t_{i}\left(\omega_{1}\right)=t_{i}\left(\omega_{2}\right)=t_{i}\left(\omega_{3}\right)=\tau_{i}$ and $\tau_{i}\left(\left\{\omega_{1}\right\}\right)=$ $\tau_{i}\left(\left\{\omega_{2}\right\}\right)=\frac{1}{2}$ and $\tau_{i}\left(\left\{\omega_{3}\right\}\right)=0$. If $\Omega=S=X$, then by Dekel, Lipman, and Rustichini (1998) the unawareness structure has trivial unawareness only. Any non-trivial partition of $X$ into separate spaces yields either no projections or violates properties (i) to (iii). We conclude that not every standard types-space with zero probability can be used to model unawareness. We understand the contribution of our work as making restrictions required for modeling unawareness precise in unawareness belief structures.

Definition 12 (Flattened Game) Given a Bayesian game with unawareness of events and (possibly) actions $\Gamma(\underline{\mathcal{S}})$, we can associate a standard Bayesian game $F(\Gamma(\underline{\mathcal{S}}))$ played on a standard type-space (with possibly allowing for varying action sets of the players across different types) in the following manner:

If $\Gamma(\underline{\mathcal{S}})=\left\langle\underline{\mathcal{S}},\left(M_{i}\right)_{i \in I},\left(\mathcal{M}_{i}\right)_{i \in I},\left(u_{i}\right)_{i \in I}\right\rangle$, where $\mathcal{S}=\left\langle\mathcal{S},\left(r_{S_{\beta}}^{S_{\alpha}}\right)_{S_{\beta} \preceq S_{\alpha}},\left(t_{i}\right)_{i \in I}\right\rangle$ is a unawareness belief structure, then set $F(\Gamma(\underline{\mathcal{S}})):=\left\langle F(\underline{\mathcal{S}}),\left(M_{i}\right)_{i \in I},\left(\mathcal{M}_{i}\right)_{i \in I},\left(u_{i}\right)_{i \in I}\right\rangle$, where 
$F(\underline{\mathcal{S}})$ is the flattened structure associated with $\underline{\mathcal{S}}$, and $\left(M_{i}\right)_{i \in I},\left(\mathcal{M}_{i}\right)_{i \in I}$, and $\left(u_{i}\right)_{i \in I}$ remain unchanged.

The flattened game is a standard Bayesian game (apart from explicitly modeling uncertainty about opponents' action sets).

Proposition 6 Since the strategy sets and the utility functions remain unchanged, we have that any strategy profile is a Bayesian equilibrium in $\Gamma(\underline{\mathcal{S}})$ if and only if it is a Bayesian equilibrium in $F(\Gamma(\underline{\mathcal{S}}))$.

The interpretation of a flattened game may be flawed in several ways. For instance, we can have types of players who are certain of their set of actions, but consider it possible that they have a larger set of actions even though they don't have a larger set of actions. This leads to serious conceptual problems, if a player were to choose such an action. A player could then "test" his own beliefs by trying to choose such actions. Consider as a simple example an unawareness belief structure with two disjoint spaces, $S_{1}=\left\{\omega_{1}\right\} \succeq S_{2}=\left\{\omega_{2}\right\}$ and one player only. Let the set of the player's available actions at $\omega_{1}$ be $\{a, b\}$, while it is just $\{a\}$ at $\omega_{2}$. The type mapping is defined by $t(\omega)\left(\left\{\omega_{2}\right\}\right)=1$ for all $\omega$. That is, although at $\omega_{1}$ the player has actions $a$ and $b$ available, he is unaware of $b$. In the flattened game, he is aware of $b$ but is certain at $\omega_{2}$ that he has just action $a$ available. But because he is aware of $b$ at $\omega_{2}$, he could try to test his belief by trying to choose $b$. The flattened game is not well-defined because it is not specified what happens if a player tries to take an action that is not available to him.

The fact that an unawareness beliefs structure can be "flattened" into a standard type spaces does not mean that unawareness has no behavior implications. Unawareness has very different properties from probability zero belief. For instance, one property that is satisfied by unawareness is symmetry (see Heifetz, Meier, and Schipper, 2013a, Proposition 5). An agent is unaware of an event if and only if she is unaware of its negation. Clearly, such a property cannot be satisfied by probability zero belief because if an agent assigns probability zero to an event, then she must assign probability one to its complement. Schipper (2013) shows that this feature captures also behavioral differences between unawareness and probability zero belief. Let's say a decision maker chooses among different contracts for buying a firm. A second contract may differ from a first contract only in a consequence for an event $E$ that is disadvantageous to the buyer. If the decision maker is indifferent between both contracts, then this is consistent with $E$ being Savage null. Yet, if the decision maker is also indifferent between the first and a third contract that differs from the first only in assigning this disadvantageous consequence to the negation of the event $E$ instead the event $E$ itself, then this behavior is inconsistent with the negation of the event $E$ or the event $E$ itself being Savage null. The decision maker behaves as if both the event $E$ and its negation are Savage null, which is impossible with probability zero belief but consistent with unawareness of the $E$ and of its negation. Thus, in models with primitives that are sufficiently rich to study decisions under unawareness, unawareness can have behavioral implications distinct from zero probability. 


\section{Further Proofs}

\section{C.1 Proof of Remark 2}

Let $\omega=\left(\alpha_{0},\left(\alpha_{i}\right)_{i \in I}\right)$ with $\alpha_{0}=\alpha$, and let $\alpha \succeq \beta \succeq \delta$. We have to show for every $i \in I^{0}$ that $\inf \left\{\alpha_{i}, \delta\right\}=\inf \left\{\delta, \inf \left\{\alpha_{i}, \beta\right\}\right\}$. Since $\delta \preceq \beta$, we have $\inf \left\{\alpha_{i}, \delta\right\} \preceq \inf \left\{\alpha_{i}, \beta\right\}$ and hence $\inf \left\{\alpha_{i}, \delta\right\} \preceq \inf \left\{\delta, \inf \left\{\alpha_{i}, \beta\right\}\right\}$. We have $\inf \left\{\delta, \inf \left\{\alpha_{i}, \beta\right\}\right\} \preceq \delta$, by definition, but since $\inf \left\{\alpha_{i}, \beta\right\} \preceq \alpha_{i}$, we also have $\inf \left\{\delta, \inf \left\{\alpha_{i}, \beta\right\}\right\} \preceq \alpha_{i}$. This implies $\inf \left\{\delta, \inf \left\{\alpha_{i}, \beta\right\}\right\} \preceq \inf \left\{\alpha_{i}, \delta\right\}$.

\section{C.2 Proof of Lemma 1}

We prove only the last claim, since the rest is obvious.

Let $\alpha \succeq \beta \succeq \delta, \omega=\left(\alpha_{j}\right)_{j \in I^{0}} \in S_{\alpha}$ and $t_{i}^{k}\left(\omega_{\beta}\right) \in \Delta\left(S_{\delta}\right)$. By definition $t_{i}^{k}(\omega) \in \Delta\left(S_{\alpha_{i}}\right)$. We have to show that $\alpha_{i} \succeq \delta$. Since $t_{i}^{k}\left(\omega_{\beta}\right) \in \Delta\left(S_{\delta}\right)$, we have by (i') that $\omega_{\beta}=\left(\beta_{j}\right)_{j \in I^{0}}$ is such that $\beta_{i}=\delta$. By the definition of $r_{\beta}^{\alpha}$, we have $\beta_{i}=\inf \left\{\alpha_{i}, \beta\right\}$. Hence $\delta \preceq \alpha_{i}$.

\section{C.3 Proof of Remark 3}

We define $\alpha \cap \beta$ to be the restricted game such that $M_{i}^{\alpha \cap \beta}:=M_{i}^{\alpha} \cap M_{i}^{\beta}$, for all $i \in I$. Note that if $\alpha, \beta \in G(\gamma)$, then $\alpha \cap \beta=\alpha \wedge \beta$ since $G(\gamma)$ is a meet-sublattice of the lattice of all restricted games.

Let $\emptyset \neq M_{i}^{\prime} \subseteq M_{i}$, where $M_{i}$ is the action set of player $i$ in $\gamma$. We have to show that $\left\{\omega \in \Omega: \mathcal{M}_{i}(\omega) \supseteq M_{i}^{\prime}\right\}$ is an event.

By definition, $G(\gamma)$ is a finite meet-sublattice of the lattice of all restricted games given $\gamma$ ordered by set inclusion of $\prod_{i \in I} M_{i}$. Fix a player $i \in I$. Recall that $\mathcal{M}_{i}(\omega)=M_{i}^{\alpha}$, for all $\omega \in S_{\alpha}$, where $M_{i}^{\alpha}$ is the action set of player $i$ in the restricted game $\alpha$.

Let $A=\left\{\alpha \in G(\gamma): M_{i}^{\alpha} \supseteq M_{i}^{\prime}\right\}$. Since $G(\gamma)$ is a finite meet-sublattice, $\bigcap_{\alpha \in A} \alpha=$ : $\alpha\left(M_{i}^{\prime}\right) \in G(\gamma)$. We have $M_{i}^{\prime} \subseteq M_{i}^{\alpha\left(M_{i}^{\prime}\right)}$ and $M_{i}^{\alpha\left(M_{i}^{\prime}\right)} \subseteq \mathcal{M}_{i}(\omega)$, for all $\omega$ such that $\mathcal{M}_{i}(\omega) \supseteq M_{i}^{\prime}$. Since for $\omega \in S_{\alpha}, \mathcal{M}_{i}(\omega)=M_{i}^{\alpha}$, we have that $\left[M_{i}^{\prime}\right]=\left\{\omega \in \Omega: \mathcal{M}_{i}(\omega) \supseteq\right.$ $\left.M_{i}^{\prime}\right\}=\left(S_{\alpha\left(M_{i}^{\prime}\right)}\right)^{\uparrow}$, which is an event.

\section{C.4 Proof of Lemma 2}

Suppose for some $i \in I, \nu_{i}$ would not be a best reply to $\nu_{-i}$. Then there exists $m_{i} \in M_{i}$ such that

$$
\varepsilon \leq v_{i}\left(m_{i}, \nu_{-i}\right)-v_{i}\left(\nu_{i}, \nu_{-i}\right)
$$


for some $\varepsilon>0$. By continuity of the utility functions in mixed strategies and in beliefs on types there exists a $k_{1}$ such that

$$
\left|v_{i}\left(\nu_{i}, \nu_{-i}\right)-U_{\left(i, t_{i}^{k}(\bar{\gamma})\right)}\left(\sigma_{i}^{k}, \sigma_{-i}^{k}\right)\right|<\frac{\varepsilon}{3}
$$

for all $k \geq k_{1}$. And likewise there exits $k_{2}$ such that

$$
\left|U_{\left(i, t_{i}^{k}(\bar{\gamma})\right)}\left(m_{i}, \sigma_{-i}^{k}\right)-v_{i}\left(m_{i}, \nu_{-i}\right)\right|<\frac{\varepsilon}{3},
$$

for all $k \geq k_{2}$.

Let $k \geq \max \left\{k_{1}, k_{2}\right\}$, then

$$
\begin{aligned}
& U_{\left(i, t_{i}^{k}(\bar{\gamma})\right)}\left(m_{i}, \sigma_{-i}^{k}\right)-U_{\left(i, t_{i}^{k}(\bar{\gamma})\right)}\left(\sigma_{i}^{k}, \sigma_{-i}^{k}\right) \\
& =\underbrace{U_{\left(i, t_{i}^{k}(\bar{\gamma})\right)}\left(m_{i}, \sigma_{-i}^{k}\right)-v_{i}\left(m_{i}, \nu_{-i}\right)}_{\geq-\frac{\varepsilon}{3}}+\underbrace{v_{i}\left(m_{i}, \nu_{-i}\right)-v_{i}\left(\nu_{i}, \nu_{-i}\right)}_{\geq \varepsilon} \\
& \quad+\underbrace{v_{i}\left(\nu_{i}, \nu_{-i}\right)-U_{\left(i, t_{i}^{k}(\bar{\gamma})\right)}\left(\sigma_{i}^{k}, \sigma_{-i}^{k}\right)}_{\geq-\frac{\varepsilon}{3}} \\
& \geq \frac{\varepsilon}{3},
\end{aligned}
$$

that is, $\sigma_{i}^{k}$ is not a best reply to $\sigma_{-i}^{k}$, for sufficiently large $k$. This implies that $\sigma^{k}$ is not an equilibrium of the game $\Gamma\left(\underline{\mathcal{S}}_{\gamma}^{k}\right)$, for sufficiently large $k$, a contradiction.

\section{C.5 Proof of Theorem 1}

For every $k=0,1, \ldots$, the Bayesian game with unawareness $\Gamma\left(\underline{\mathcal{S}}_{\gamma}^{k}\right)$ has an equilibrium by Corollary 1. Since the set of mixed strategy combinations at $\bar{\gamma}$ is a closed and bounded subset of an Euclidean space, the sequence of equilibria $\left(\sigma^{k}(\bar{\gamma})\right)_{k=0}^{\infty}$ has a subsequence that converges to some $\nu$. By Lemma 2, $\nu$ is an unawareness perfect equilibrium of $\tilde{\gamma}$. $\square$

\section{C.6 Proof of Theorem 2}

" $\Rightarrow$ ": Let $\nu$ be an unawareness perfect equilibrium of $\tilde{\gamma}$. Then there exists a sequence of Bayesian games with unawareness $\left(\Gamma\left(\underline{\mathcal{S}}_{\gamma}^{k}\right)\right)_{k=0}^{\infty}$ with corresponding Bayesian Nash equilibria $\sigma^{k} \in E\left(\Gamma\left(\underline{\mathcal{S}}_{\gamma}^{k}\right)\right), k=0,1, \ldots$, for which $\sigma^{k}(\bar{\gamma}) \rightarrow \nu$ as $k \rightarrow \infty$.

From Pearce (1984, Lemma 4) follows that a Nash equilibrium profile $\nu$ of the original game $\tilde{\gamma}$ is undominated if and only if for every $i \in N, \nu_{i}$ is a best response to a completely mixed strategy profile of opponents in the original game $\tilde{\gamma}$. Note that by Property $(\mathrm{v})$, $\sum_{\omega \in S_{\gamma}} t_{i}^{k}(\bar{\gamma})(\{\omega\}) \sigma_{-i}^{k}(\omega)$ is equivalent to a completely mixed strategy profile of opponents for any $k=0,1, \ldots$, in the original game $\tilde{\gamma}$. Since $\sigma_{i}^{k}(\bar{\gamma}) \rightarrow \nu_{i}$, for any $m_{i} \in M_{i}$ with $\nu_{i}\left(m_{i}\right)>0$, there exists a sufficiently large $k\left(m_{i}\right)$ such that for all $k \geq k\left(m_{i}\right), \sigma_{i}^{k}(\bar{\gamma})\left(m_{i}\right)>$ 
0. Since $M_{i}$ is finite, there is a $k_{\max }$ such for all $k \geq k_{\max }, \sigma_{i}^{k}(\bar{\gamma})\left(m_{i}\right)>0$ for all $m_{i} \in M_{i}$ with $\nu_{i}\left(m_{i}\right)>0$. Thus, any such $m_{i} \in M_{i}$ is a best reply to $\sum_{\omega \in S_{\gamma}} t_{i}^{k}(\bar{\gamma})(\{\omega\}) \sigma_{-i}^{k}(\omega)$, for all $k \geq k_{\max }$. Hence, $\nu_{i}$ is a best reply to the completely mixed belief equivalent to $\sum_{\omega \in S_{\gamma}} t_{i}^{k_{\max }}(\bar{\gamma})(\{\omega\}) \sigma_{-i}^{k_{\max }}(\omega)$.

"६": Recall that from Pearce (1984, Lemma 4) it follows that a Nash equilibrium profile $\nu$ is undominated in the original game $\tilde{\gamma}$ if and only if for every $i \in N, \nu_{i}$ is a best response to a completely mixed strategy profile of opponents $\tilde{\nu}_{-i}$ in $\tilde{\gamma}$. We will use these completely mixed strategy profiles $\tilde{\nu}_{-i}, i \in I$, to construct a sequence of Bayesian games with unawareness $\left(\Gamma\left(\underline{\mathcal{S}}_{\gamma}^{k}\right)\right)_{k=0}^{\infty}$ with Bayesian Nash equilibria $\sigma^{k} \in E\left(\Gamma\left(\underline{\mathcal{S}}_{\gamma}^{k}\right)\right), k=0,1, \ldots$, for which $\sigma^{k}(\bar{\gamma}) \rightarrow \nu$ as $k \rightarrow \infty$.

Given the augmented strategic game $\gamma$, consider any restricted strategic game $\alpha$ with $d_{i} \in M_{i}^{\alpha}$ for all $i \in I$ and $1 \leq\left|M_{i}^{\alpha}\right| \leq 2$. Note that by construction in any such restricted game, each player has at most one non-default action and if there is a non-default action for player $i$, then this non-default action is the strict dominant Nash equilibrium action of $\alpha$. Let $G(\gamma)$ be the set comprising of all such games, $\perp$, and $\gamma$ itself. Note that $G(\gamma)$ is rich (see page 16) and a lattice.

We now construct a sequence of Bayesian games with unawareness of actions by defining for each player $i \in I$ the type mapping as follows: For $m_{-i} \in \tilde{M}_{-i}$, define $\ell\left(m_{-i}\right)$ to be the number of profiles $\left(\alpha_{j}\right)_{j \in I \backslash\{i\}}$ with $\alpha_{j} \in G(\gamma) \backslash\{\perp, \gamma\}, j \in I \backslash\{i\}$, for which $\left\{m_{-i}\right\}=\prod_{j \in I \backslash\{i\}}\left(M_{j}^{\alpha_{j}} \backslash\left\{d_{j}\right\}\right)$. (Note that in such a profile we have $\left|M_{j}^{\alpha_{j}}\right|=2$, for all $j \in I \backslash\{i\})$.

Fix $\varepsilon \in(0,1)$. If $\omega=\left(\alpha_{j}\right)_{j \in I^{0}}$ with $\alpha_{j} \in G(\gamma) \backslash\{\perp, \gamma\}$ such that $\left|M_{j}^{\alpha_{j}}\right|=2$, for $j \in I \backslash\{i\}$ and $\alpha_{j}=\gamma$ for $j \in\{0, i\}$, let $t_{i}^{k}(\bar{\gamma})(\{\omega\})=\frac{\varepsilon^{k}}{\ell\left(m_{-i}\right)} \tilde{\nu}_{-i}\left(m_{-i}\right)$ with $\left\{m_{-i}\right\}=$ $\prod_{j \in I \backslash\{i\}}\left(M_{j}^{\alpha_{j}} \backslash\left\{d_{j}\right\}\right)$.

All the remaining probability mass of player $i$ 's type at state $\bar{\gamma}$ at $k, 1-\varepsilon^{k}$, is assigned to $t_{i}^{k}(\bar{\gamma})(\{\bar{\gamma}\})$. We impose properties (i'), (ii'), and (iv'). Property (iii') is implied by Lemma 1. Properties (v) and (vi) are satisfied by construction. Beliefs of player $i$ in states different from $\bar{\gamma}$ are then either completely determined by beliefs in $\bar{\gamma}$ via properties (ii') and (iv') or can be assigned so as to satisfy properties (i') to (vi).

Next, we construct a sequence of Bayesian Nash equilibria whose limit in state $\bar{\gamma}$ is the undominated Nash equilibrium $\nu$ of the original game $\tilde{\gamma}$. For any player $i$ and any $k$, let $\sigma_{i}^{k}(\omega)=\nu_{i}$ for all $\omega \in S_{\gamma}$ with $\omega=\left(\beta_{j}\right)_{j \in I^{0}}$ such that $\beta_{i}=\gamma$. Moreover, set $\sigma_{i}^{k}(\omega)\left(d_{i}\right)=1$ in any other state $\omega=\left(\beta_{j}\right)_{j \in I^{0}}$ with $M^{\beta_{i}}=\left\{d_{i}\right\}$. Finally, set $\sigma_{i}^{k}(\omega)\left(m_{i}\right)=1$ with $m_{i} \neq d_{i}$ in any state $\omega=\left(\beta_{j}\right)_{j \in I^{0}}$ with $M^{\beta_{i}}=\left\{m_{i}, d_{i}\right\}$. (Recall that such a non-default action is strictly dominant.) Since in the latter states, $\sigma_{i}^{k}(\omega) \in \Delta\left(M_{i}^{\alpha}\right)$, for $\alpha \prec \gamma$, we extend $\sigma_{i}^{k}(\omega)$ to $\tilde{\sigma}_{i}^{k}(\omega) \in \Delta\left(M_{i}\right)$ by setting $\tilde{\sigma}_{i}^{k}(\omega)\left(m_{i}\right):=\sigma_{i}^{k}(\omega)\left(m_{i}\right)$ for all $\omega \in \Omega_{\gamma}$ and $m_{i} \in M_{i}^{\alpha}$.

Note that $\sum_{\omega \in S_{\gamma}} t_{i}^{k}(\bar{\gamma})(\{\omega\}) \tilde{\sigma}_{-i}^{k}(\omega) \rightarrow \nu_{-i}$ for $k \rightarrow \infty$. Moreover, for any $k$, $\sum_{\omega \in S_{\gamma}} t_{i}^{k}(\bar{\gamma})(\{\omega\}) \tilde{\sigma}_{-i}^{k}(\omega)$ is a convex combination of $\tilde{\nu}_{-i}$ and $\nu_{-i}$.

To see that $\sigma^{k}$ is a Bayesian Nash equilibrium for any $k=0,1, \ldots$, note that $\sigma_{i}^{k}(\omega)=\nu_{i}$ with $\omega=\left(\beta_{j}\right)_{j \in I^{0}}$ such that $\beta_{i}=\gamma$ is a best reply to $\nu_{-i}$ since $\nu$ is Nash equilibrium of $\tilde{\gamma}$. 
Since $\nu$ is an undominated Nash equilibrium of $\tilde{\gamma}$, we also noted above that $\nu_{i}$ is a best reply against $\tilde{\nu}_{-i}$. Hence $\nu_{i}$ is also a best reply to any convex combination of $\nu_{-i}$ and $\tilde{\nu}_{-i}$. It follows that for any $k=0,1, \ldots, \sigma_{i}^{k}$ is a Bayesian Nash equilibrium mixture of player $i$ in the Bayesian game with unawareness $\Gamma\left(\underline{\mathcal{S}}_{\gamma}^{k}\right)$. Since $\sigma_{i}^{k}(\bar{\gamma})=\nu_{i}$ for all $k$ and $i \in I, \nu$ is an Unawareness perfect equilibrium of $\tilde{\gamma}$.

\section{References}

[1] Čopič, J. and A. Galeotti (2006). Awareness as an equilibrium notion: Normal-form games, mimeo.

[2] Dekel, E., Lipman, B., and A. Rustichini (1998). Standard state-space models preclude unawareness, Econometrica 66, 159-173.

[3] Feinberg, Y. (2009). Games with unawareness, Stanford GSB.

[4] Feinberg, Y. (2005). Games with incomplete awareness, Stanford GSB.

[5] Gilboa, I. and D. Schmeidler (1994). Additive representations of non-additive measures and the Choquet integral, Annals of Operations Research 52, 43-65.

[6] Grabiszewski, K. (2013). On the rejectability of the subjective expected utility theory, ITAM.

[7] Grant, S. and J. Quiggin (2013). Inductive reasoning about unawareness, Economic Theory, forthcoming.

[8] Halpern, J. and L. Rêgo (2008). Interactive unawareness revisited, Games and Economic Behavior 62, 232-262.

[9] Halpern, J. and L. Rêgo (2013). Extensive games with possibly unaware players, Mathematical Social Sciences, forthcoming.

[10] Harsanyi, J. (1967/68). Games with incomplete information played 'Bayesian' players, Part I, II, and II, Management Science 14, 159-182, 320-334, 486-502.

[11] Heifetz, A., Meier, M., and B.C. Schipper (2013a). Unawareness, beliefs, and speculative trade, Games and Economic Behavior 77, 100-121.

[12] Heifetz, A., Meier, M., and B.C. Schipper (2013b). Dynamic unawareness and rationalizable behavior, Games and Economic Behavior 81, 50-68.

[13] Heifetz, A., Meier, M., and B.C. Schipper (2012). Unawareness, beliefs, and speculative trade, University of California, Davis.

[14] Heifetz, A., Meier, M., and B.C. Schipper (2011b). Prudent rationalizability in generalized extensive-form games, University of California, Davis. 
[15] Heifetz, A., Meier, M., and B.C. Schipper (2008). A canonical model for interactive unawareness, Games and Economic Behavior 62, 304-324.

[16] Heifetz, A., Meier, M., and B.C. Schipper (2006). Interactive unawareness, Journal of Economic Theory 130, 78-94.

[17] Heinsalu, S. (2013). Universal type spaces with unawareness, Yale University.

[18] Hu H. and H. W. Stuart, Jr. (2001). An epistemic analysis of the Harsanyi transformation, International Journal of Game Theory 30, 517-525.

[19] Li, J. (2006b). Dynamic games with perfect awareness information, mimeo.

[20] Meier, M., and B.C. Schipper (2012). Conditional dominance in games with unawareness, University of California, Davis.

[21] Meier, M. and B.C. Schipper (2009). Speculative trade under unawareness - The infinite case, University of California, Davis.

[22] Mertens, J.-F. and S. Zamir (1985). Formulation of Bayesian analysis for games with incomplete information, International Journal of Game Theory 14, 1-29.

[23] Modica, S. and A. Rustichini (1999). Unawareness and partitional information structures, Games and Economic Behavior 27, 265-298.

[24] Modica, S. and A. Rustichini (1994). Awareness and partitional information structures, Theory and Decision 37, 107-124.

[25] Nash, J. (1950). Equilibrium points in n-person games, Proceedings of the National Academy of Sciences 36, 48-49.

[26] Nielsen, C. and A. Sebald (2011). Unawareness in dynamic psychological games, University of Copenhagen.

[27] Ozbay, E. (2007). Unawareness and strategic announcements in games with uncertainty, in: Samet, D. (ed.), Proceedings of the 11th conference on Theoretical Aspects of Rationality and Knowledge, Presses Universitaires de Louvain, pp. 231238.

[28] Pearce, D. (1984). Rationalizable strategic behavior and the problem of perfection, Econometrica 52, 1029-1050.

[29] Peleg, B., Potters, J., and S.H. Tijs (1996). Minimality of consistent solutions for strategic games, in particular for potential games, Economic Theory 7, 81-93.

[30] Peleg, B. and S.H. Tijs (1996). The consistency principle for games in strategic form, International Journal of Game Theory 29, 13-34. 
[31] Rêgo, L. and J. Halpern (2012). Generalized Solution Concepts in Games with Possibly Unaware Players, International Journal of Game Theory 41, 131-155.

[32] Sadzik, T. (2006). Knowledge, awareness and probabilistic beliefs, mimeo.

[33] Schipper, B.C. (2013). Awareness-dependent subjective expected utility, International Journal of Game Theory 42, 725-753.

[34] Selten, R. (1975). Reexamination of the perfectness concept for equilibrium points in extensive games, International Journal of Game Theory 4, 25-55.

[35] Van Damme, E. (1991). Stability and perfection of Nash equilibria, Berlin et al.: Springer-Verlag. 